\title{
COLLECTION UNDERSTANDING
}

\author{
A Thesis \\ by \\ MICHELLE T. CHANG \\ Submitted to the Office of Graduate Studies of \\ Texas A\&M University \\ in partial fulfillment of the requirements for the degree of \\ MASTER OF SCIENCE
}

May 2004

Major Subject: Computer Science 


\title{
COLLECTION UNDERSTANDING
}

\author{
A Thesis \\ by \\ MICHELLE T. CHANG \\ Submitted to Texas A\&M University \\ in partial fulfillment of the requirements \\ for the degree of \\ MASTER OF SCIENCE
}

Approved as to style and content by:

\begin{tabular}{c}
\hline $\begin{array}{c}\text { John J. Leggett } \\
\text { (Chair of Committee) }\end{array}$ \\
\hline $\begin{array}{c}\text { Richard Furuta } \\
\text { (Member) }\end{array}$
\end{tabular}

\begin{tabular}{c}
\hline $\begin{array}{c}\text { James Rosenheim } \\
\text { (Member) }\end{array}$ \\
\hline $\begin{array}{c}\text { Valerie E. Taylor } \\
\text { (Head of Department) }\end{array}$
\end{tabular}

May 2004

Major Subject: Computer Science 


\author{
ABSTRACT \\ Collection Understanding. (May 2004) \\ Michelle T. Chang, B.S., Angelo State University \\ Chair of Advisory Committee: Dr. John J. Leggett
}

Collection understanding shifts the traditional focus of retrieval in large collections from locating specific artifacts to gaining a comprehensive view of the collection. Visualization tools are critical to the process of efficient collection understanding. By presenting simple visual interfaces and intuitive methods of interacting with a collection, users come to understand the essence of the collection by focusing on the artifacts. This thesis discusses a practical approach for enhancing collection understanding in image collections. 


\section{ACKNOWLEDGMENTS}

Special thanks to Dr. John J. Leggett for his unending guidance, support and encouragement in completing this thesis.

I am also indebted to Dr. Randolph G. Bias, Patrick J. Williams and Samuel A. Burns for conducting an independent evaluation of the Collection Understanding Tool.

I extend gracious appreciation to Dr. Andruid Kerne for his insightful comments and his work on Collage Machine which has influenced my research. In addition, I would like to thank Dr. Richard Furuta for his interpretation of this work.

Thanks also to Dr. Enrique Mallen for providing the image collection of the Online Picasso Project. 


\section{TABLE OF CONTENTS}

Page

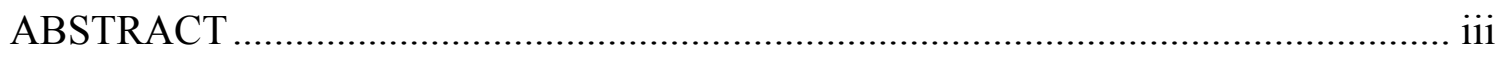

ACKNOWLEDGMENTS................................................................................

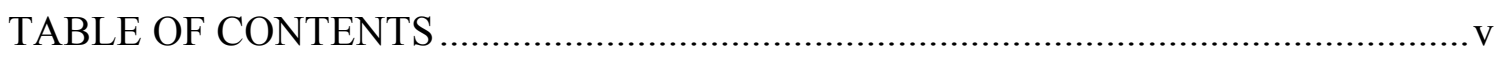

LIST OF FIGURES.................................................................................... vii

INTRODUCTION TO COLLECTION UNDERSTANDING ...................................... 1

COLLECTION UNDERSTANDING VS. INFORMATION RETRIEVAL ................... 3

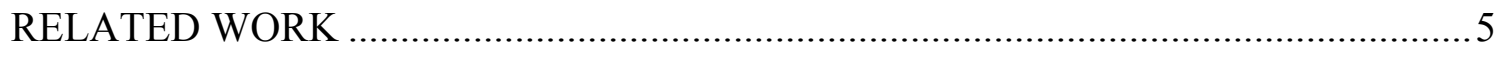

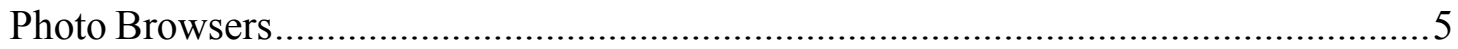

Time as Essence for Photo Browsing ........................................................ 5

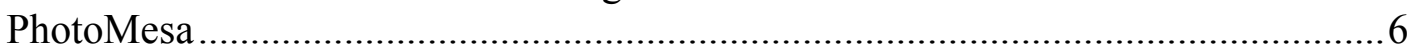

PhotoFinder ................................................................................................. 7

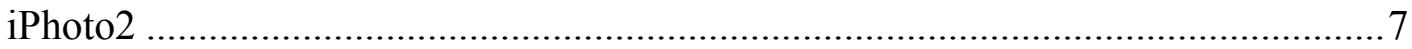

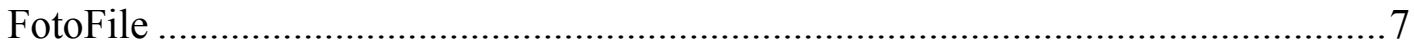

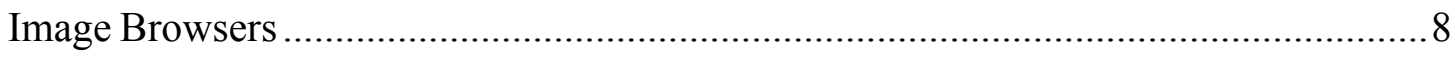

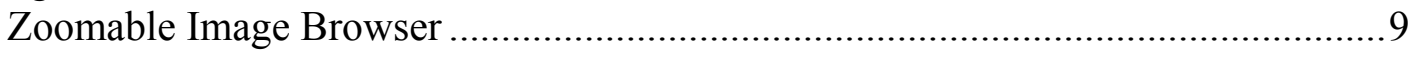

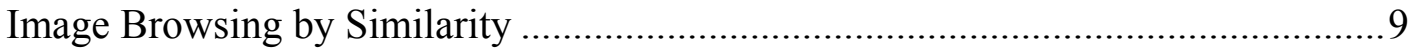

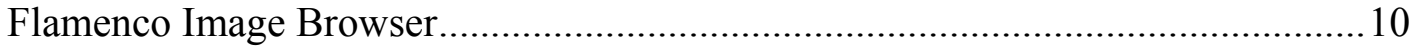

Vmedia JPEG 2000 Interactive Image Browser............................................ 10

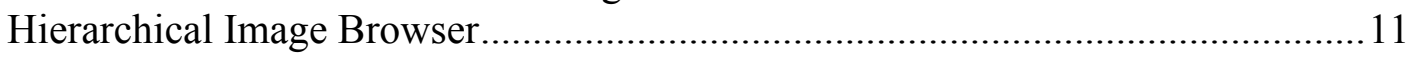

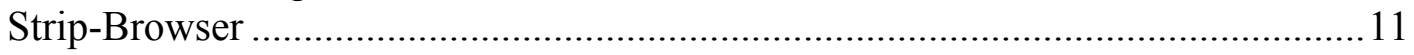

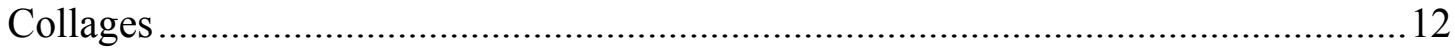

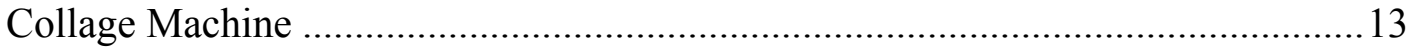

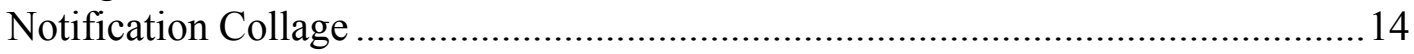

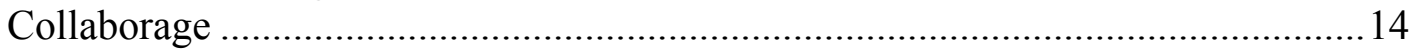

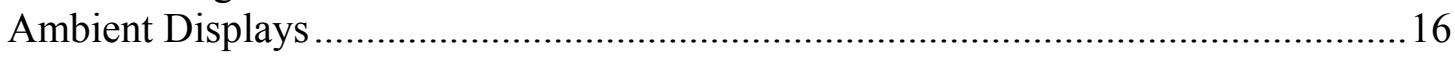

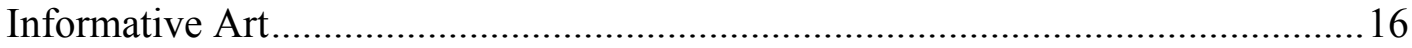

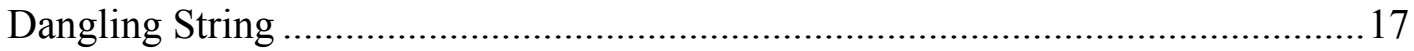

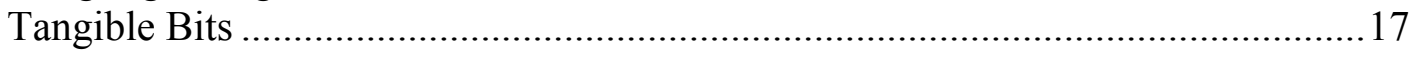

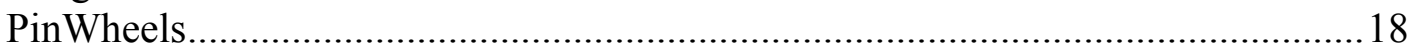

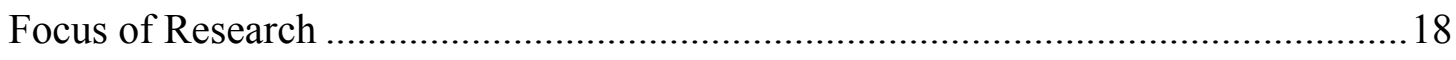




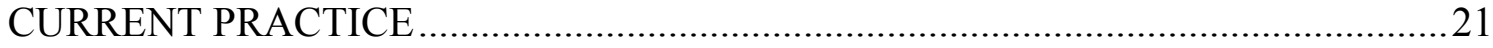

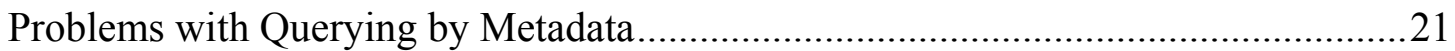

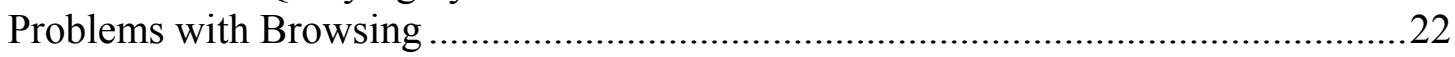

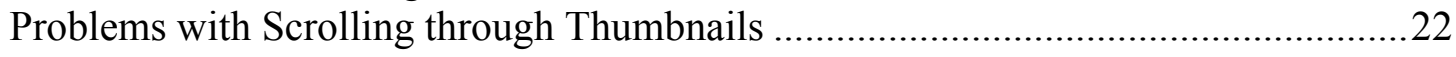

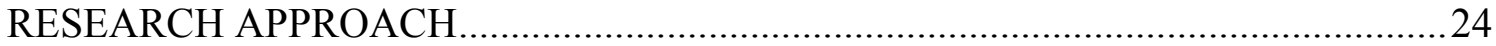

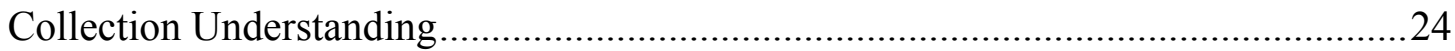

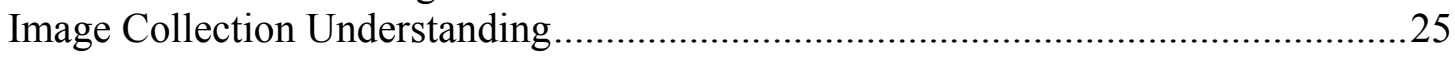

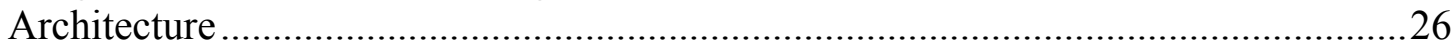

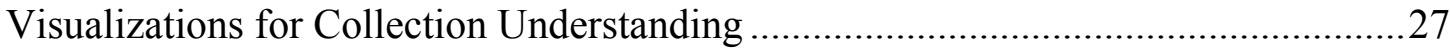

Using Streaming Collage for Collection Understanding....................................28

Using an Ambient Slideshow for Collection Understanding .................................. 37

Using Variably Gridded Thumbnails for Collection Understanding .......................39

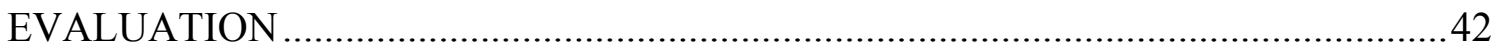

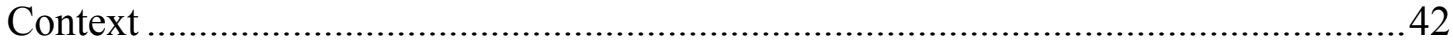

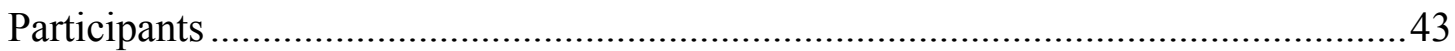

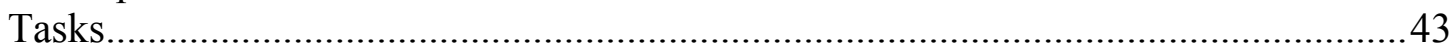

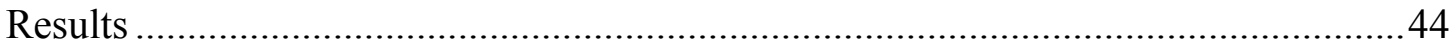

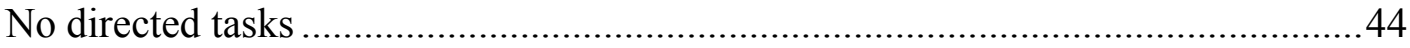

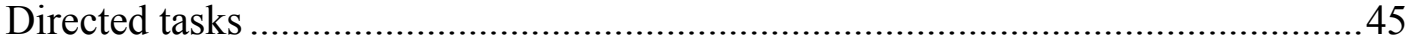

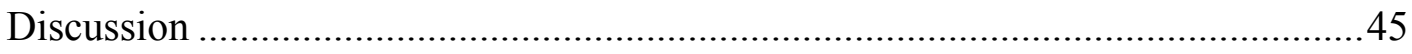

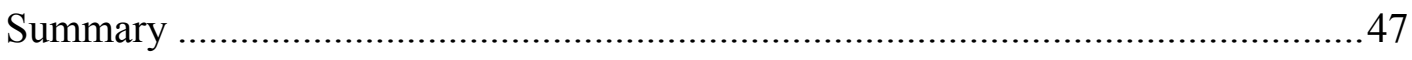

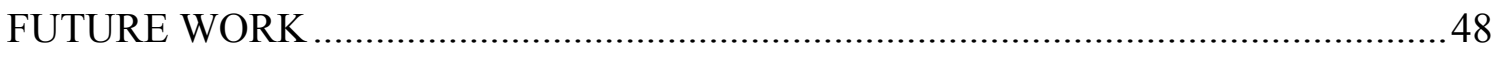

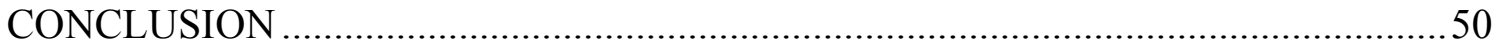

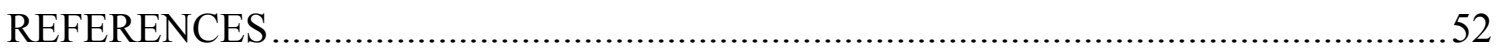

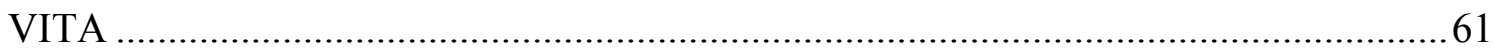




\section{LIST OF FIGURES}

Figure 1. System overview...............................................26

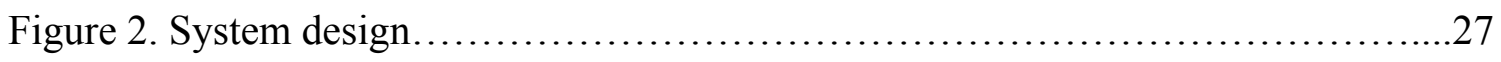

Figure 3. Using streaming collage for understanding the Online Picasso Collection......29

Figure 4. Metadata filtering on the Online Picasso Collection.........................31

Figure 5. Creating a personal Picasso collection................................33

Figure 6. Deleting image titled "Corbeille de fruits"...............................34

Figure 7. Confirmation dialog for deleting image titled "Corbeille de fruits".................35

Figure 8. Selecting the menu option "Save to my SubCollection"......................36

Figure 9. Ambient Picasso................................................ 38

Figure 10. Variably gridded thumbnails for the Online Picasso Collection.............40 


\section{INTRODUCTION TO COLLECTION UNDERSTANDING}

Digital libraries are providing novel means of information access to large collections of artifacts. Widely available digital imaging technologies have resulted in a dramatic increase in the number of collected artifacts. However, these large image collections pose a challenge for the collection provider when trying to convey the actual collection's contents. Users cannot effectively understand what constitutes a large image collection if they are unable to see what is in the collection in an efficient manner.

Visualization tools are critical to the process of efficient collection understanding, not only for viewing the artifacts but also for understanding their metadata and content. By presenting simple visual interfaces and intuitive methods of interacting with a collection, users come to understand the essence of the collection by focusing on the artifacts.

Various methods have been used to express information about desired artifacts in large image collections [Witten et al. 1999]. These image retrieval methods include browsing using metadata [Elliott 2001; Witten et al. 2000; Yee et al. 2003], keyword/phrase searching on specific metadata fields [Witten and Bainbridge 2002], finding similar images through feature extraction such as contour, texture [Malik et al. 2000], shape and color [Ong et al. 2002], and text overlaid on video [Christel et al. 2002]. Directly searching on these collections traditionally returns pages of surrogates

This thesis follows the style and format of Association for Computing Machinery Transactions on Computer-Human Interaction. 
that serve as multimedia abstractions for previewing the artifacts. Users may browse through these surrogates and select specific ones to see additional information (assigned metadata). These methods help users to find particular artifacts in the collection.

Collection understanding has a different focus when viewing the collection. The goal here is to understand the entire collection by comprehending the "whole as a sum of the (relationships among the) parts." By "parts," we mean a subset of artifacts in the collection. The user should be able to iteratively specify subsets, visualize the resulting collection artifacts easily and derive their own understanding.

Screen space is a key factor in designing visualization tools for any collection. Displays are limited with respect to the number of artifacts that may be shown without scrolling or navigating to the next page of results. Scrolling is slow and navigating becomes very tedious for the user [Zhai et al. 1997]. When the representative artifacts are separated and cannot be seen simultaneously on the same display, users become distracted from their main goal of collection understanding. When the result set is very large, users may find the process of examining the artifacts almost impossible, since they must traverse each page of surrogates. This thesis is exploring various techniques, including streaming collage [Kerne 1997; 2000; 2001; 2002a; 2002b], variably gridded thumbnails [Combs and Bederson 1999; Rodden et al. 2001], and ambient slideshows [Mankoff et al. 2003], to more effectively use the screen space. By utilizing visualization techniques that eliminate manual scrolling and the process of browsing, collection understanding becomes the central goal. 


\section{COLLECTION UNDERSTANDING VS. INFORMATION RETRIEVAL}

Information retrieval (IR) technologies are traditionally used to enable users to find specific artifacts. Users must be able to define queries by specifying values for metadata fields. IR interfaces facilitate this "find the needle in the haystack" approach.

Collection understanding is, in some sense, in direct opposition to the IR approach. The users may have no prior knowledge of the metadata fields or values. Users first acquaint themselves with the actual artifacts and then come to understand what the metadata fields and values mean and how the collection artifacts vary based on dynamically generated criteria. Instead of having a pre-conceived notion of what artifacts to look for in the collection, users take an exploratory approach by defining their own metadata filters and forming their own structures for viewing the artifacts. Users may or may not have a plan for what artifacts they are looking for in a collection but once they have experienced or acquired a general sense of the collection by metadata filtering and information visualization, they are better able to understand how metadata and artifacts interplay in forming the collection, and hence match their filtering criteria to the current situation.

Additionally, users are often faced with the dilemma of defining an artifact in an IR interface. Users have a general idea of what they are looking for but can neither define the condition(s) using IR terms nor express a specific description. Users are compelled to find a representation of their query in a form that can be understood by a system they find difficult and unnatural to use. Collection understanding facilitates a visual illustration of the artifacts over time and gives insight about how the collector 
chose to describe the artifacts using metadata. Users gradually learn the collector's choice of metadata and values by experience through interaction with the contents of the collection. 


\section{RELATED WORK}

While collection understanding is a general idea, our initial work focuses on image collections. Several means of visualizing image collections have been explored in previous research [Borner and Chen 2002; Card et al. 1999] and this area is currently very active in the digital libraries research community [Furnas 1986; Ong et al. 2002; Shneiderman et al. 2000; Witten et al. 2000]. This research project draws most heavily from the following four areas: photo browsers, image browsers, collage, and ambient displays.

\section{Photo Browsers}

Photo browsers manage collections of photographs. They typically include home photo collections and introduce browsing techniques for novice users [Kang and Shneiderman 2000]. They allow users to easily digitize and organize their photographs into categories of events, based on metadata created by the user [Bederson 2001; Kuchinsky et al. 1999], file names and directories [Bederson 2001], and the date and time [Graham et al. 2002] that the photos were taken.

\section{Time as Essence for Photo Browsing}

Graham, et al. (Stanford) developed two photo browsers, Calendar Browser and Hierarchical Browser [Graham et al. 2002]. These browsers automatically grouped photographs based on the time-stamp of the digital images. Instead of using metadata to relate photographs in the collection, both browsers used the time stamps and organized 
pictures based on year, month, day and time, as well as aperture settings, distance from the focal plane, and whether a flash was used. Photos are viewed by using time to narrow the search for images. Since photos from a particular event are taken roughly around the same time, the browsers automatically index these images using clustering techniques to represent "bursts" of times as separate events. The Calendar Browser uses a calendar metaphor to display the images, while the Hierarchical Browser uses the metaphor of a Windows File Explorer or Macintosh Finder utility.

\section{PhotoMesa}

Work done by Bederson at the Human-Computer Interaction Laboratory, University of Maryland automatically groups images together for display using folder, year and month [Bederson 2001]. PhotoMesa uses two algorithms: Quantum Treemaps and Bubblemaps. This photo browser organizes pictures in a two-dimensional grid, where images sharing directory location, similarity in time, or a shared word in their filename are placed together. Multiple hierarchical directories of thumbnails are placed side by side on the browser window, and various zooming magnifications are used to present the actual images to the user. Clustering of images is also done by metadata that may be available on the file system. PhotoMesa does not depend solely on this metadata to organize its images. When user-generated metadata is not available, date and time are used to cluster the photographs. 


\section{PhotoFinder}

Research done on PhotoFinder by Shneiderman at the Human Computer Interaction Lab, University of Maryland, allows users to organize images into "collections" when they selectively choose one image to represent the entire collection [Kang and Shneiderman 2000]. The interface consists of all of these representative images. Selecting one image will display the entire collection as a grid of thumbnail images. PhotoFinder displays the full-sized image in a separate pane within the interface, thereby curbing the problem of managing multiple windows. Also, to help in the retrieval of photos, an interface for searching the database is provided.

iPhoto2

Developed by Apple [Apple iPhoto2 2003], iPhoto2 is a commercial image manager similar to PhotoMesa in that users automatically index pictures without requiring annotations. Images are grouped by "rolls" and a batch of photos on a CD is uploaded to the computer and stored as one collection, providing a means of filing groups of photos for easy browsing. Images are displayed using a two-dimensional grid of thumbnails.

FotoFile

FotoFile [Kudhinsky, et al. 1999] combines both human and automatic annotation methods to organize collections of images. A friendly interface for users is provided to manually annotate content using pre-defined metadata attributes such as creation date, location, subject, people, title, and description [Dublin Core 1998]. This 
metadata is used to define the collection's hierarchical image management. Users query the collection using Boolean operations or ranked searches based on feature extraction. A photo album is used as the metaphor to display the images. Basically, this system provides a two-dimensional grid of thumbnails of varying sizes with the title of the photo appearing above the thumbnails. In addition, FotoFile allows users to tell a story through arrangement of photos into groups to build more dynamic storytelling structures. Time and face recognition are used to implement the automatic annotation feature. Once a name has been entered for a particular photo, this system tries to match other faces to that name, thereby automatically generating annotations for the photo. The user must confirm the system's annotations before they are associated with the images. FotoFile also visualizes the collection by borrowing the Hyperbolic Tree package from Inxight Software. However, problems have arisen in usability studies when the outer-rim photos in the hyperbolic tree grouped well and users assumed they belonged to the same category when, in fact, the images were clustered based on color distribution and texture.

\section{Image Browsers}

Image browsers manage a more diverse collection of artifacts, including

photographs [Kang and Shneiderman 2000], architectural drawings [Elliott 2001; 2002] and museum paintings [Bjork 2000]. They provide effective ways to retrieve artifacts and to improve user interfaces for viewing these collections [Bjork 2000; Furnas 1986; Rodden et al. 2001]. 
Zoomable Image Browser

Bederson developed Zoomable Image Browser (ZIB) at the University of Maryland [Bederson 2001]. ZIB integrates image browsing and image retrieval. A search area is provided for both simple search and advanced search using Boolean operators. Images satisfying the query are returned in the Browse section of the interface as thumbnails arranged across the screen. ZIB differs from other browsing systems in that the user has control of the number of images displayed and the resolution of the images. Users can pan and zoom individual images and can see various magnifications of the image. When users want to look at a few of the images in detail, they zoom in on the view to acquire larger and higher resolution images. As a result, fewer images are shown on the display. Successive searches produce a history of the user's browsing and all previous queries are displayed next to the search form. This history interface also includes a snapshot of the images returned from the history query.

Image Browsing by Similarity

Rodden et al. at the University of Cambridge investigated similarity-based arrangements for displaying image thumbnails [Rodden et al. 2001]. Organization by similarity changes the default ordering of these thumbnails and arranges them according to their mutual similarity. Similarity is measured using textual captions from the images, global image properties such as color and texture, and the broad spatial layout of the image region. The cosine coefficient measure and the vector space model [Witten et al. 1999] are used to calculate similarity. The goal is to help users quickly locate a given 
image or group of images. The images are displayed on the screen in three spatial arrangements: multi-dimensional scaling with overlap, a 12 × 12 grid and a $10 \times 10$ grid.

\section{Flamenco Image Browser}

Ame Elliott at the University of California, Berkeley developed a prototype system to allow architects to access a large on-line image collection of architectural drawings [Elliott 2001]. The collection has at least nine metadata fields, storing the location, architect, style, kind of building, etc. Images are queried on these metadata fields and the system returns images together with suggestions of alternative terms to broaden or refine queries. Flamenco (FLexible Access to MEtadata in NOvel Combinations) presents an interface with four different regions. Each region shows images that share a metadata field value associated with the query, thereby sorting the image results into four different but related categories. Users browse the collection by changing the kind of metadata in each region, allowing the architect to expand or shrink the query [Elliott et al. 2002]. Thus, the interface provides hints to guide the user to the next query.

\section{Vmedia JPEG 2000 Interactive Image Browser}

Li, et al. at Microsoft Research China developed an interactive image browser to help Internet users efficiently browse large images [Li and Sun 2000]. These highresolution images are slow to download over the Internet, often require high bandwidth, and are unsuitable for handheld devices such as PDAs. JPEG 2000 compresses the image and renders a scalable coarse quality image into fixed sized blocks. When the 
lower resolution image is transmitted, the Vmedia (Virtual media) image browser allows the user to select a region of interest (ROI) from the fixed-size blocks. Once a ROI is selected, a lossless ROI is then transmitted and shown within ten seconds, creating a faster and more pleasing browsing experience.

\section{Hierarchical Image Browser}

Bjork at the Interactive Institute, Sweden examined the Flip Zooming technique to present images of paintings and sculptures [Bjork 2000]. Flip zooming places several items in parallel, so users can clearly see the items together and compare them. These image items represent a dynamically generated information structure based on the classification the user chooses for organizing the images. When a user selects a particular image as the focus, this image is enlarged and presented clearly while simultaneously showing the overall information structure containing the other images. This type of visualization resembles a "fisheye" view [Furnas 1986], where a focus plus context visualization [Baudisch and Good 2002] is provided to the user. When applied to painting collections, artwork from different sections of the museum can be viewed on the same display, providing a context for the visualization [Baldonado and Winograd 1997]. A selected image becomes enlarged enabling focus. Enlarging images from different parts of the visualization enables comparison among different schools of art.

\section{Strip-Browser}

van Liere, et al. at the Center for Mathematics and Computer Science, CWI, The Netherlands [van Liere and de Leeuw 1999] created a filmstrip interaction metaphor for 
viewing images. The filmstrip consists of a set of cells, each holding a thumbnail image. The browser uses a virtual reality interface to present the query results as a series of filmstrips that all fit within one screen. Interaction with the filmstrip is done by interpreting the users' head movements. Detection of user's movements is accomplished by using immersive, stereoscopic displays and six-degrees-of-freedom position trackers. When users move their heads towards the screen, an enlarged image is shown. Users browse the images at a certain speed by looking at the filmstrip at an angle. This angle is measured between the user's line of sight and the perpendicular line to the strip-browser display. The larger the angle, the faster the images will display. Additionally, the user's line of sight determines which filmstrip is being examined. Using Strip-Browser, users quickly grasp the content of a subset of images and easily focus their attention on new images by using head movements.

\section{Collages}

Collage is an effective tool for utilizing screen space, allowing a large collection of images to be viewed simultaneously. Collage has been used to provide a novel web browsing technique [Kerne 1997; 2000; 2001], form aesthetic and dynamic art pieces [Fogarty et al. 2001], create a group awareness tool [Greenberg and Rounding 2001], and show a physical information collage connected to an electronic information display [Moran et al. 1999]. 


\section{Collage Machine}

Andruid Kerne (Texas A\&M University) created Collage Machine as a new form of web visualization [Kerne 1997; 2001; 2002b]. The collage interface saves the user from downloading webpages, scanning the content of the entire document and clicking on links to find pertinent websites. In addition, users typically rely on the first few webpages returned by a search engine, which limits their view of many other relevant webpages. Collage Machine is initiated when several search terms or URLs are input into the program to seed the search engine. The application then crawls the web and extracts media elements, such as text and images from webpages, presenting them as a collage in real time [Kerne 2002a]. Users can change the arrangement of the media elements to redefine the direction of the collage. Collage Machine learns which media is interesting to the user based on the selection and placement of images, hence the Collage Machine becomes an agent of recombination [Kerne 2001]. The "Positive Grab" tool expresses interest in similar media elements, while the "Negative Grab" tool expresses disinterest in similar media elements. Selecting the "cut" tool represents an extreme form of disinterest. Clicking on a media element opens a new window with the web page containing the selected element.

Chance procedures are used to create the collage, thus no collage is a replica of another [Kerne 1997]. The media elements are re-contextualized on the collage screen. That is, the context of the collage changes in two ways: streaming media elements create a new presentation environment on the screen, and the elements are juxtaposed and 
hence associated in different ways [Kerne 2001]. This change in context becomes the motivation for deriving new patterns and meaning.

\section{Notification Collage}

Greenberg, et al. (University of Calgary) created Notification Collage, which uses the collage technique for their groupware system [Greenberg and Rounding 2001]. Colleagues post media elements such as video, images, text messages, graphs, sticky notes, slide shows, and desktop screenshots on a collaborative bulletin board. Notification Collage hopes to create new awareness and collaboration among its community members. People become engaged in the elements shown by this Notification Collage, allowing greater opportunity for conversation and interaction. Visual elements in Notification Collage are randomly positioned, with newer items always being placed on top.

\section{Collaborage}

Moran, et al. (Xerox Palo Alto Research Center) developed applications such as the "In/Out and Away Board" and "Project Task Wall" [Moran et al. 1999]. A collaborage is a collaborative collage that maps physically represented information on a surface to electronic information. This electronic representation is then made visible using browsers to promote group awareness and contact. The In/Out and Away Board enables group members to indicate their current physical location to other members. When they leave the office, group members place their badge, which has their name, picture and a glyph tag, on a physical board. This physical board consists of three 
columns (In, Out, Comment). A group member places the badge in either the In or Out column and optionally writes a comment. The Collaborage system tracks this activity, takes snapshots of the Comments column, and logs the information in a database. A CGI script then reads the database and displays this information on web browsers for other group members to view. Similarly, Project Task Wall allows group members to keep track of the group's progress by logging in active tasks posted on the wall. Collaborage is an input device which links a physical wall visible in only one location to a widely accessible electronic display in a browser.

\section{Aesthetic Information Collages}

Collages have the potential to form aesthetic and dynamic art pieces, as described by Fogarty et al. (Carnegie Mellon University) [Fogarty et al. 2001]. Typically, the primary function of an information display is to convey information, which may be aesthetically interesting. However, the Kandinsky system is designed to create aesthetically pleasing collages as the primary goal, with the ability to convey information as the secondary goal. That is, these collages convey information as an added bonus, thus reversing the typical role of an information display. Just as a painting for the home or the office is purchased for its aesthetic appeal, the Kandinsky system can be used to generate an aesthetic display. Artists define an aesthetic template for the Kandinsky system to follow. An aesthetic template is a set of aesthetic principles, such as the dominant colors of the display and the overall compositional form. The Kandinsky system uses these aesthetic properties to determine the visual appeal of the display. 


\section{Ambient Displays}

Ambient displays do not require a user's undivided attention or continuous realtime interaction. Rather, they display non-critical information slowly in the background environment [Mankoff et al. 2003]. Through chance encounters, they engage the user's attention without requiring direct focus. Ambient displays have been used in slow technology informative art (where aesthetic displays update themselves slowly) [Holmquist and Skog 2003], the dangling string [Weiser and Brown 1995], pinwheels [Ishii et al. 2001], and tangible bits [Ishii and Brygg 1997].

\section{Informative Art}

Holmquist et al. defines the concept of informative art as a method for integrating information visualization in the everyday environment [Holmquist and Skog 2003]. Electronic information displays will soon form an integral part in ubiquitous computing through the use of flat panel LCD displays, giving rise to the future of visual art displays. For instance, these decorative art displays can show weather composition in six different cities by altering the size of a square on a display, where each square represents a particular city and the size of the square reflects the degree of heat in the city. Similarly, global earthquake activity can be displayed as "landscape art". The image of a lawn, which represents the world map, is shown on the information display. When an earthquake occurs, an image of a stone is placed on the lawn. Similar to weather composition, the size of the stone corresponds to the magnitude of the earthquake. Thus, these informative art pieces do not represent applications that convey exact information but are rather meant to act as a decorative art installation. 


\section{Dangling String}

Natalie Jeremijenko created the "Dangling String" - an eight-foot piece of plastic string hanging from an electric motor in the ceiling [Weiser and Brown 1995]. The electric motor is connected to an Ethernet cable so that bits of information flowing through the network cause a tiny twitch in the motor. A large flow of bits causes the string to swivel rapidly with a characteristic noise, whereas a low traffic of bits causes the string to twitch slowly. These intangible bits therefore become visible, audible and tangible. The information depicted engages the user's attention both in the periphery and as the center of attention.

\section{Tangible Bits}

Ishii, et al. designed three systems, metaDESK, transBOARD and ambientROOM, to reduce the gap between cyberspace and the physical environment and to make digital information tangible [Ishii and Brygg 1997]. Users are allowed to touch, see, "grasp and manipulate" the bits when they are coupled with architectural surfaces. Users of metaDESK and transBOARD move physical objects to manipulate bits in the center of attention (foreground) whereas ambientROOM focuses on the periphery of the user's attention (background). In metaDESK, icons are replaced by physical icons (phicons), which are small physical models on a desk surface representing for instance, buildings on a map. Hence the user visually and haptically interacts with architectural space. The transBOARD is a networked physical whiteboard, which allows distributed users over the Internet to record drawing activity using physical pens and erasers with a 
scanning infrared laser. Drawing sessions are stored on a web server to be accessed at a later time. ambientROOM uses light, shadow, sound, airflow and waterflow to communicate subtle information in the user's periphery attention [Ishii et al. 1998]. For example, webpage activity can be mapped to a steady rainfall where each hit to the webpage causes steady raindrops. The rainfall remains in the periphery of the user's attention until a change occurs such as when the rain suddenly stops.

PinWheels

Ishii, et al. developed a Pinwheel installation consisting of 40 computercontrolled pinwheels in a museum context [Ishii et al. 1998; 2001]. The pinwheel units spin in a "wind of bits" based on the speed of information flow from cyberspace. This invisible information flow from a local network or the Internet represents a new form of information visualization that reduces the gap between the digital and physical world. In addition, users become aware of and connected to others' status and activities in the Pinwheel ambient display.

\section{Focus of Research}

Past work in image browsing tools provide mechanisms for the user to actively search for a particular image in the collection. However, the focus here is different in that we are not trying to retrieve specific images. Instead, we want to understand the relationships among the images in the entire collection so that we have a more comprehensive understanding of the whole collection and its contents. In addition, most 
of the applications discussed above display a scrolling list of thumbnails across and down the screen.

Current work in IR interfaces also try to give participants a starting point for finding and browsing artifacts in the collection. The Flamenco image browser [Elliott 2001; 2002; Yee et al. 2003] allows users to dynamically view metadata values using explicit exposure of hierarchically faceted metadata [Elliott 2002]. Thus, users do not feel lost when they are given metadata feedback in the current query state. However, Flamenco focuses on externalizing the metadata to improve IR search. Therefore participants spend a great deal of time learning about metadata word descriptions of the collection and do not see (in their mind's eye) the actual artifacts of the collection. As participants become exposed to the other faceted metadata, they must still click on the links, scroll and page through thumbnails to view the actual contents that constitute the collection.

The "berry-picking" model described by Bates [Bates 1989] suggests ways to improve IR searches. According to Bates, IR searches are static one-time views and the results are a single output set. In the berry-picking model, participants use an incremental method for probing information where they learn how to refine a query based on previous result sets. With iterative feedback, users successively improve their search criteria by evaluating the efficacy of former searches. The final results are obtained from a series of incremental adjustments based on new insight found along the way to create an emergent search. However, Bates' model still focuses on improving probe searches. Although her model recognizes incremental learning based on previous 
results, collection understanding isn't about finding specific artifacts but rather, coming to understand the essence of the whole collection.

Streaming collage, variably gridded thumbnails and ambient slideshows are introduced in this thesis as different mechanisms for visualizing image collections. For example, through the use of streaming collage and metadata filtering to present image elements, users can derive their own conclusions from the collection of images. 


\section{CURRENT PRACTICE}

Three main problems plague current digital library interfaces for querying large image collections: querying by metadata is ambiguous, browsing is predefined and inflexible, and scrolling through artifacts becomes burdensome.

\section{Problems with Querying by Metadata}

While creating metadata is time-consuming for collectors, querying metadata is currently the most commonly used method for accessing images. Users enter a metadata query and matching images (or their surrogates) are returned from the collection. However, the user is limited with respect to how the creator/maintainer defines the metadata. In addition, most metadata descriptions are vague. They contain few sentences and provide little information about the possible values that satisfy the metadata field. Instead, users are left to guess how the creator categorized the metadata and cannot query the image collection unless they know what is in the collection. This process of trying to decide how to begin a search is inefficient and hampers the users' intention to learn about the collection.

If the user is successful at deriving the values for the metadata fields, the query results generated are specific to the metadata that the user entered and the user never sees similar artifacts in the collection that share some of the metadata values. Relationships among the image elements and the metadata are lost. The user obtains results based on knowing what the metadata values are, but doesn't gather any 
information about the remainder of the collection, losing the real benefit of understanding the entire collection.

\section{Problems with Browsing}

Browsing is another popular technique for image access but browsing categories are usually pre-defined and static, forcing the user to follow a fixed structure with little flexibility for adapting to the user's needs. The user simply "points and clicks", narrowing the collection to a subset of images. This technique is very time-consuming and may involve a large amount of pointing and clicking to get to the actual images.

Collection understanding is not focused on narrowing the view of a collection to a small subset of images. Instead of trying to find specific images, we would like the user to start with some image(s), and then expand/narrow that view to more/fewer images from the collection, gradually deriving relationships about the complete collection. Users progressively learn about the collection through dynamic interaction with artifacts and metadata.

\section{Problems with Scrolling through Thumbnails}

When images are returned from a query, they are typically displayed using thumbnails across and/or down the screen [Combs and Bederson 1999; Furnas 1986; Kang and Shneiderman 2000]. Users scroll through the results and choose images of interest. With limited screen space, the user must scroll through these images or click the next page link to see more results. With either technique, the previous image matches are 
no longer in view and the user cannot see the entire result set. Thus, image viewing becomes a limiting task and the collection is more difficult to understand. 


\section{RESEARCH APPROACH}

With increasingly inexpensive mass storage, there is immense motivation to digitize movies, images, books, magazines and newspapers. Compression techniques are used to compress and store these documents efficiently and allow for quick retrieval [Witten et al. 1999]. The challenge is to filter the most relevant artifacts of information and present them in a useful manner to the user. Text-based retrieval associated with image collections certainly narrows the results of a user's query and provides faster response time when searching for a particular artifact. However, the indexing and image retrieval problem has already been solved by quick hashing techniques and is not the focus of this research. We are not trying to find a more reliable technique for locating certain images in a large collection. Rather, we are trying to create visualization tools to understand the collection as an entity. Existing digital library interfaces do not provide adequate mechanisms for collection understanding. More perceptive mechanisms are needed to simplify the view of large image collections, allowing users to efficiently grasp the essence of the collection.

\section{Collection Understanding}

Collection understanding is an attempt to view the artifacts that constitute the collection in an extensive sense. We are trying to learn about the general underlying theme of the collection, the features the elements have in common and the varying qualities that link them as a collection. By increasing our awareness and obtaining a comprehensive outlook, we hope to appreciate the collection builder's (or curator's) 
point of view about what the collection represents and what characteristics the image elements share as members of the collection, so that we may derive our own perception of the collection. Intuitive interfaces are needed that allow users to efficiently grasp the essence of the collection.

\section{Image Collection Understanding}

Typical access to image collections consists of querying metadata fields and being presented with responses that match the query. These metadata fields are descriptive fields that characterize the image such as time, title, photographer, medium, dimension and event [Dublin Core 1998]. They represent structured information to help manage the images in the collection and to facilitate image description and discovery [Witten et al. 2000]. However, results that match metadata are specific to the query and give no broad perspective as to what the collection actually contains. For instance, what are the actual artifacts in the collection? What determines whether an artifact belongs to this collection or not? What are the relationships among images that form a subset of the collection?

The curator can provide collection-level metadata describing the motivation for this collection, why the images were chosen for the collection, how the images were collected, and the factors that came into play to decide that these images should be collected together. However, this general descriptive metadata does not answer the question pertaining to the actual artifacts contained in the collection and it gives no concrete visual depiction of how sub-collections are related via various metadata values. 
Users should be able to see in their mind's eye what the artifacts are and vary the metadata values dynamically to see what subset of the collection would be returned.

Currently, this research is exploring the following three visualization techniques for image collection understanding: streaming collage, ambient slideshows and variably gridded thumbnails.

\section{Architecture}

Figure 1 gives a high-level system overview of the proposed architecture.

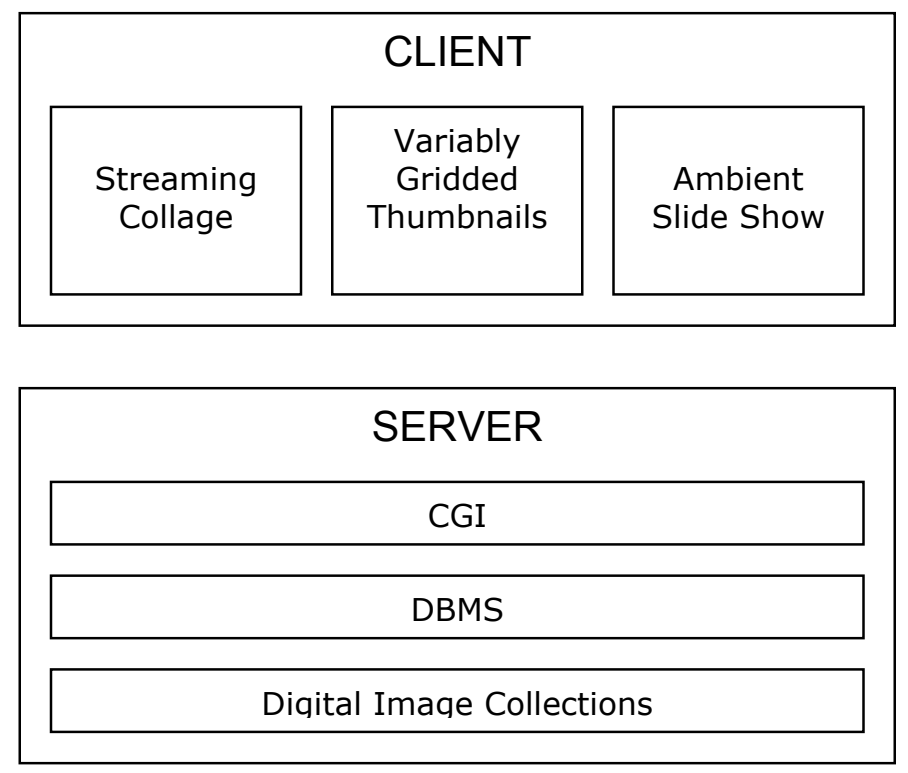

Figure 1. System overview.

Figure 2 gives a high-level system design for the proposed interaction among the client, database and server programming environments. Java and JavaScript facilitate client 
access to the image collections, which are stored in a MySQL database on the server side. Perl CGI scripts are used to access the database and return results to the client using a Java-CGI bridge.

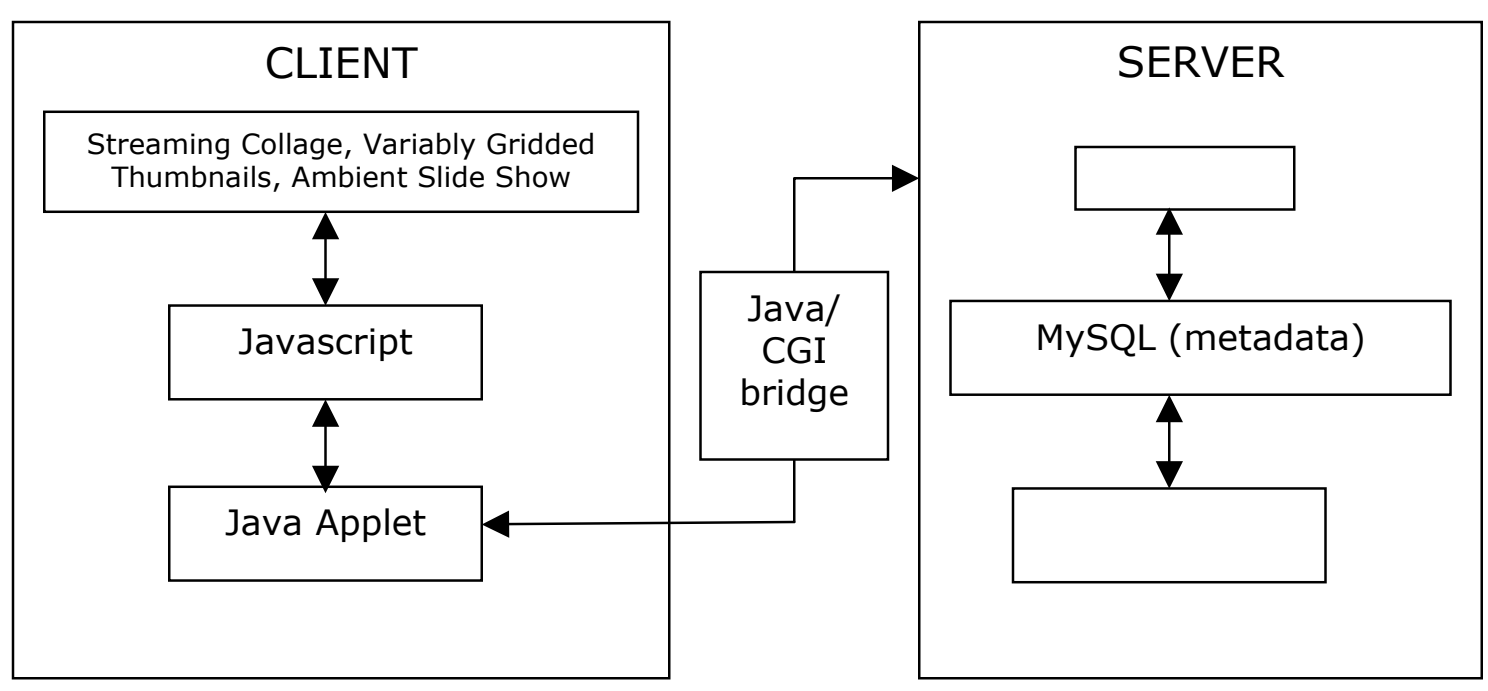

Figure 2. System design.

\section{Visualizations for Collection Understanding}

Interaction with the three visualization techniques for collection understanding: streaming collage, ambient slideshows and variably gridded thumbnails are elaborated below. The Online Picasso Project [Mallen 2004] (a collection of Picasso's life and works, consisting of approximately 7,000 images, from 1881-1973) was used as the image test collection. 


\section{Using Streaming Collage for Collection Understanding}

The literal meaning of collage is "glued stuff" [Kerne 2001]. According to the Merriam-Webster Online Dictionary, a collage is a work created by the combination of materials from different sources [Merriam Webster 2004]. The artist's purpose in making collage is to evoke some idea or feeling from the audience based on the content of the collage and to invite observers to create their own associations and interactions among the elements that constitute the collage. By streaming collage, we refer to the changing content of the collage - the images are dynamically displayed so that the collage is constructed interactively in time [Kerne 2001].

Through streaming collage, users become aware of the images that constitute the general collection and their relationships. Figure 3 shows a streaming collage prototype. On the left, a window collages the images in real time and on the right, a metadata filtering form shows the metadata fields corresponding to the collage. Streaming collage places images in the window using the metadata from the form, making the best use of limited screen resources. 


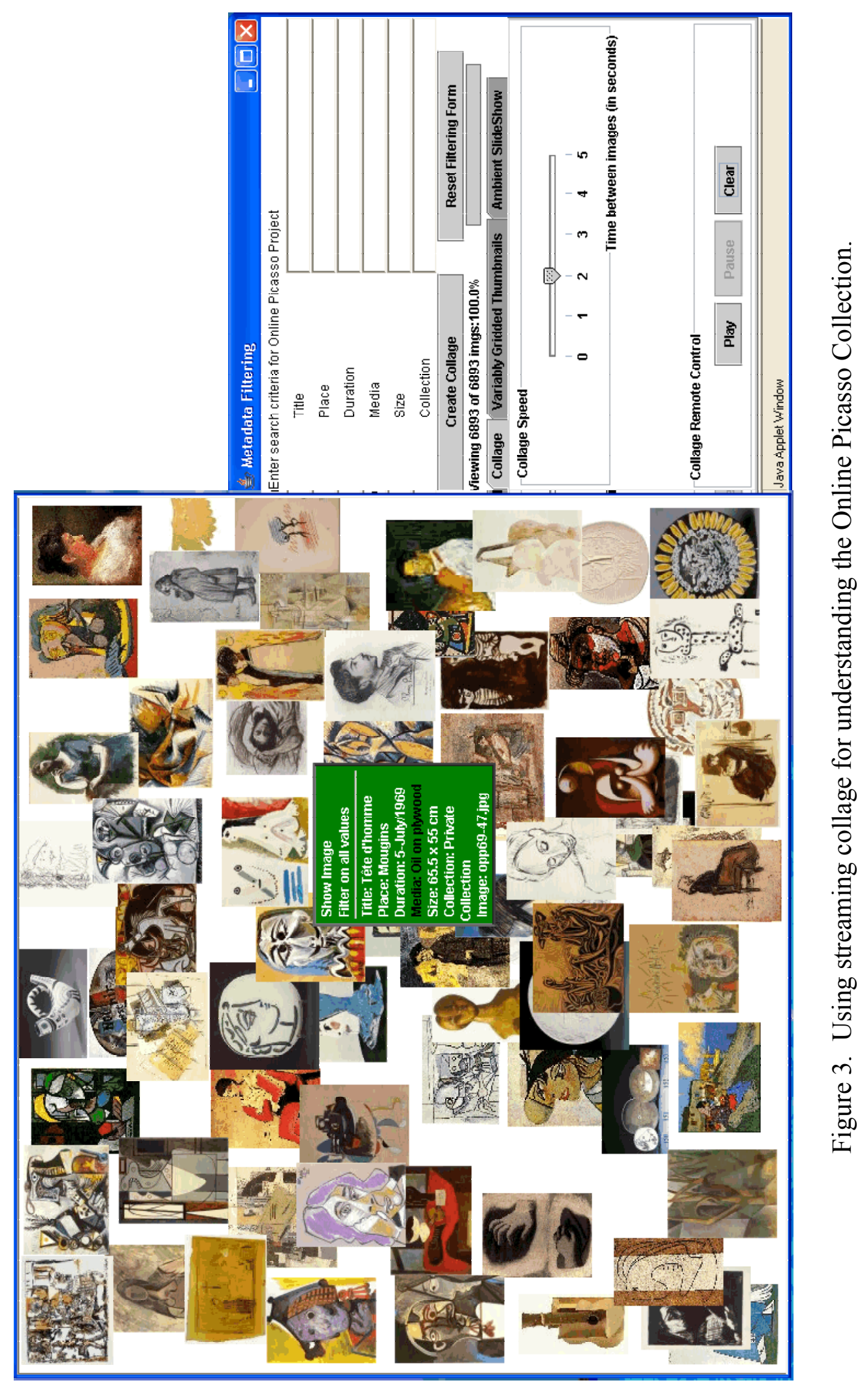


Using streaming collage as the visualization mechanism, the user can then use the metadata filtering form or the collage display to adjust metadata values to see how those changes affect the results of the collage. We refer to this process as metadata filtering - by gradually removing or adding values for the metadata fields, users come to a better understanding of the collection [Baldonado and Winograd 1997].

A user begins the process of collection understanding using either the entire collection (as in Figure 3) or specific images as the starting point. In the first case, the user may be visiting the image collection for the first time, perhaps not knowing any metadata fields or values. When the user selects the "Create Collage" option, the entire collection randomly collages on the display. Once the user spots interesting image elements, the metadata values are displayed to the user with a right-click of the mouse (Figure 3).

The user may then select any of these fields to further filter the metadata, showing more images sharing this metadata value or, at the user's choice, not sharing this metadata value. Figure 4 shows the results of filtering the collection on the media metadata field for the value of "oil on plywood". 


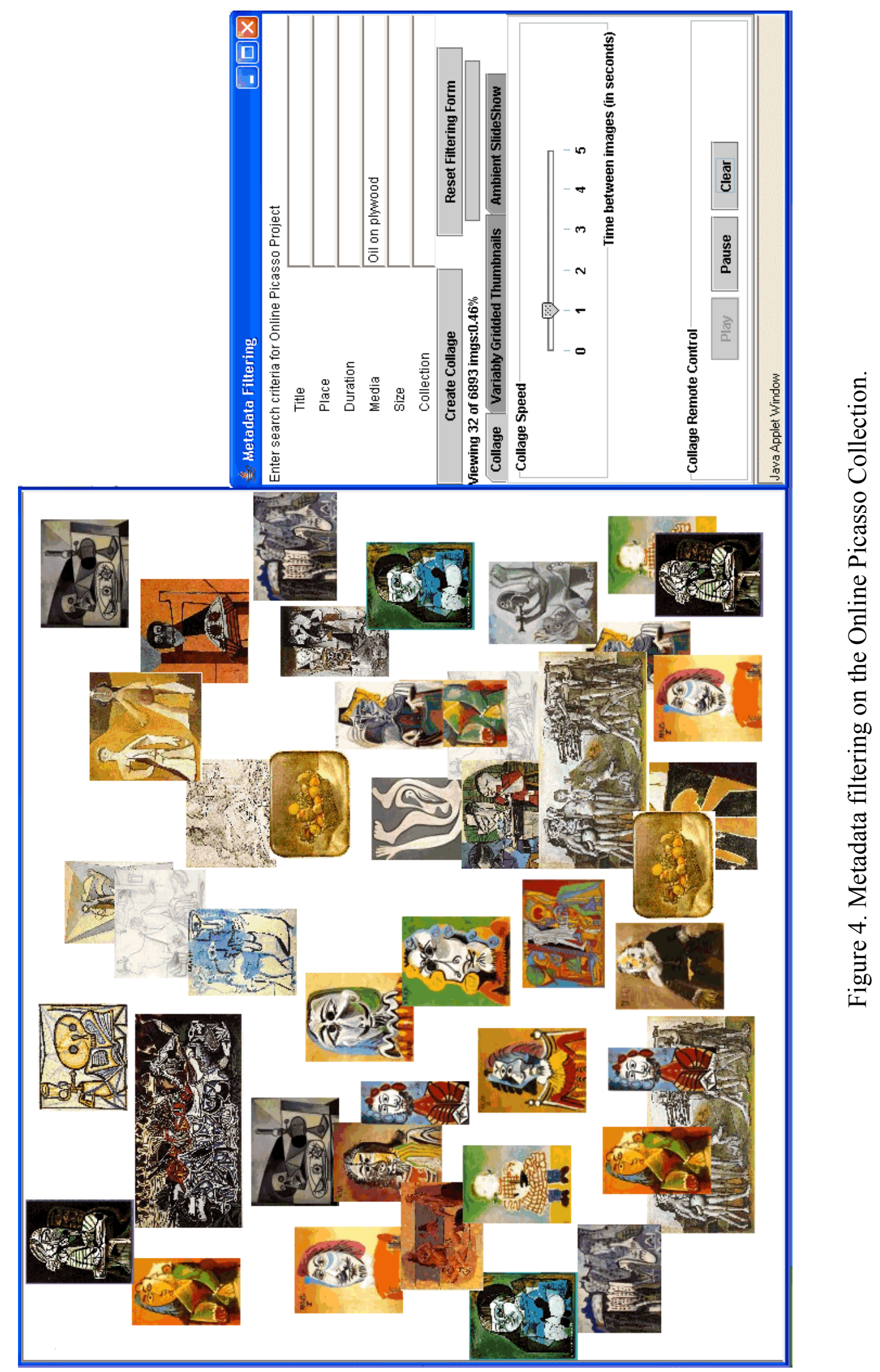


In the second case, users begin with a subset of the collection sharing certain metadata values. From this subset of the collection, users may choose to either narrow or expand the image subset by dynamically changing the metadata values as they increasingly come to a better understanding of the collection. In both cases, the dynamically generated results are shown on the collage display. Additionally, users can select higher-resolution images of the thumbnails for further examination in a subcollection window by selecting the "Show Image" item on the right-click menu (Figure 5).

The sub-collection window has two menu options which allow users to delete images previously added or to save images to personal subcollections. Selected images have a black border and are selected with a left-click of the mouse. Continuously leftclicking on an image toggles the border on and off. In Figure 6, the image titled "Corbeille de fruits" is selected from the sub-collection. When the "Delete selected images" menu option is chosen, or the "Del" key is pressed, a confirmation dialog box appears to confirm deletion of the selected images (Figure 7). Additionally, choosing the "Save to my Subcollection" menu option saves the sub-collection of images to an HTML file (Figure 8). 


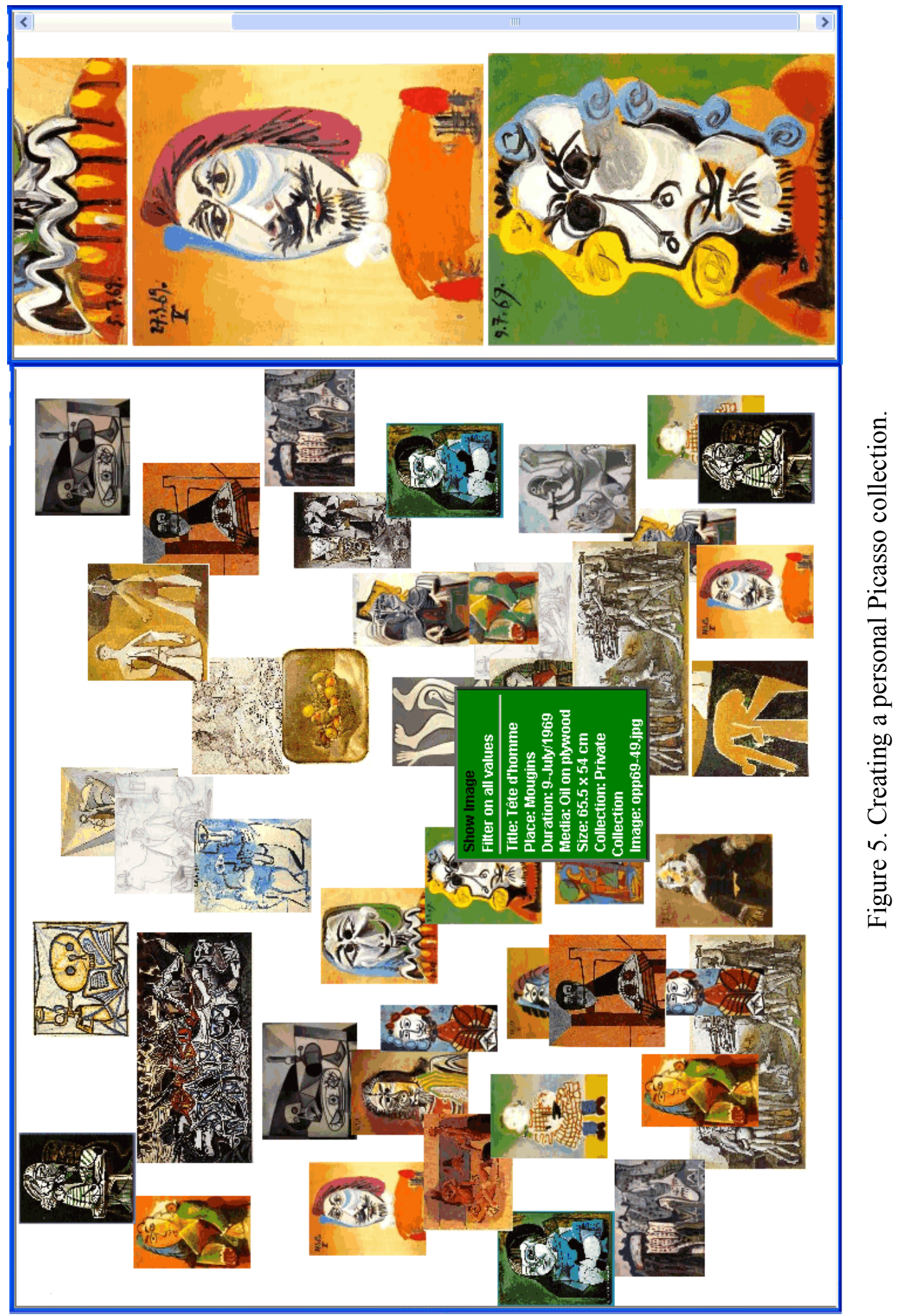




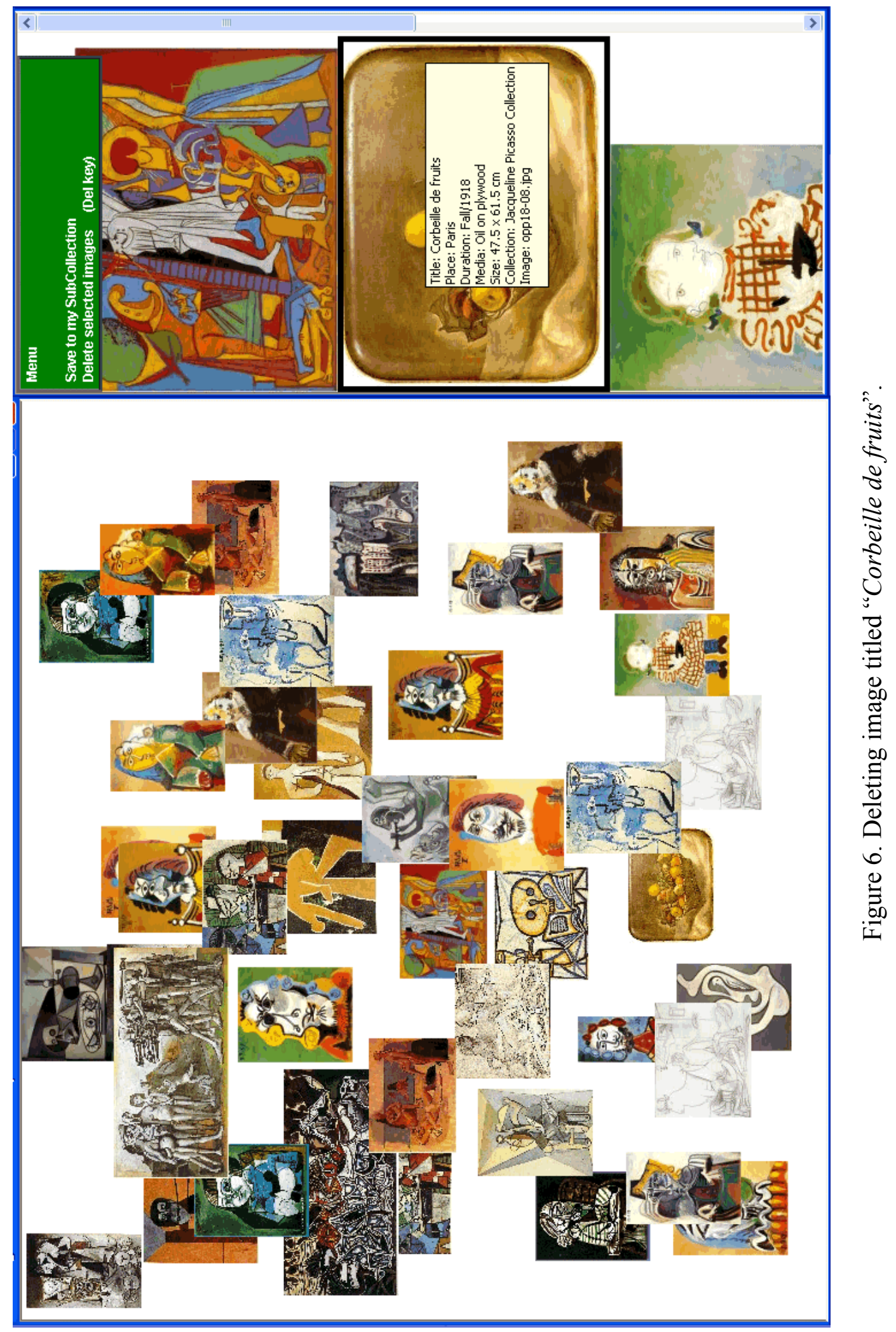




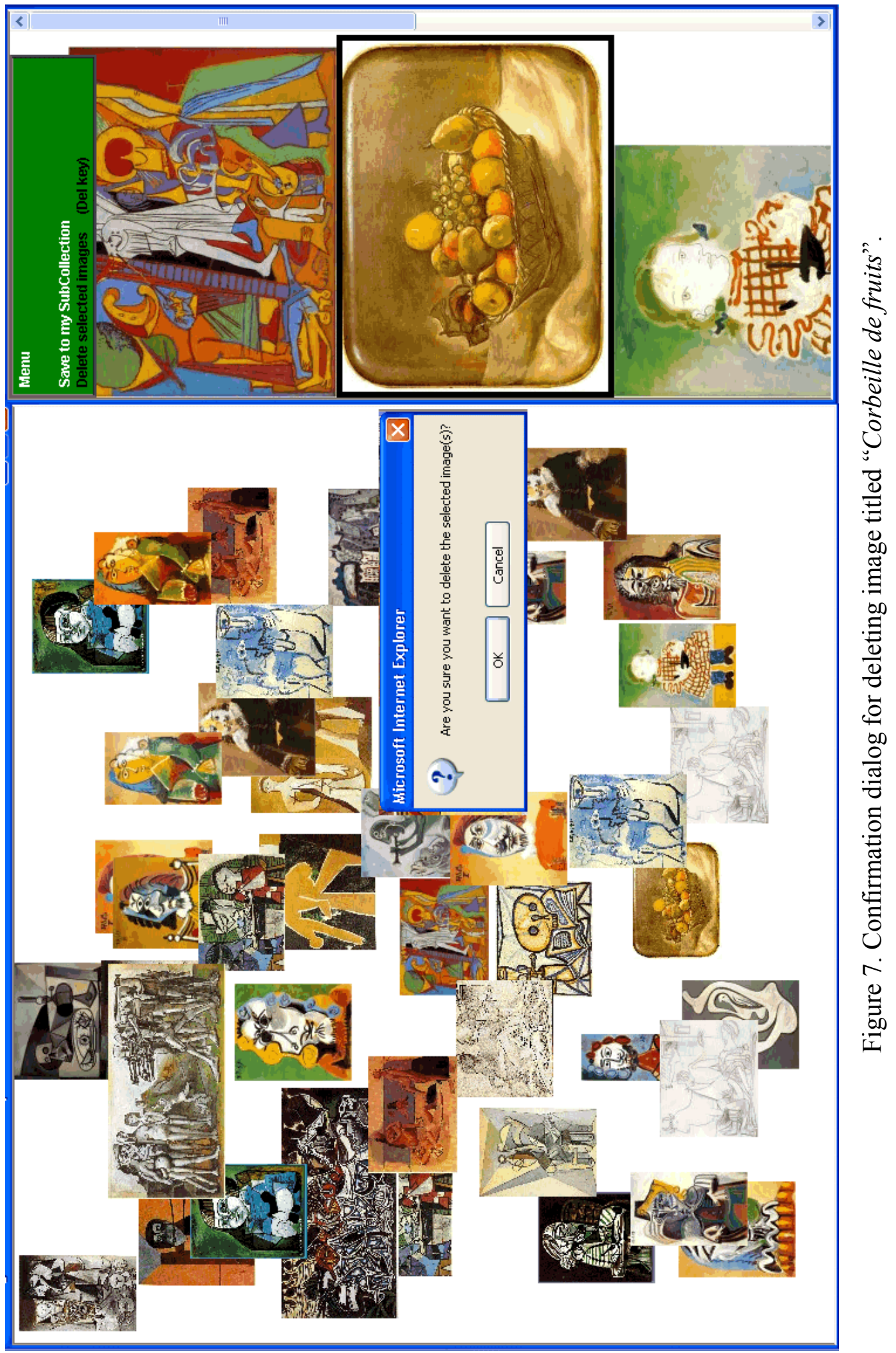




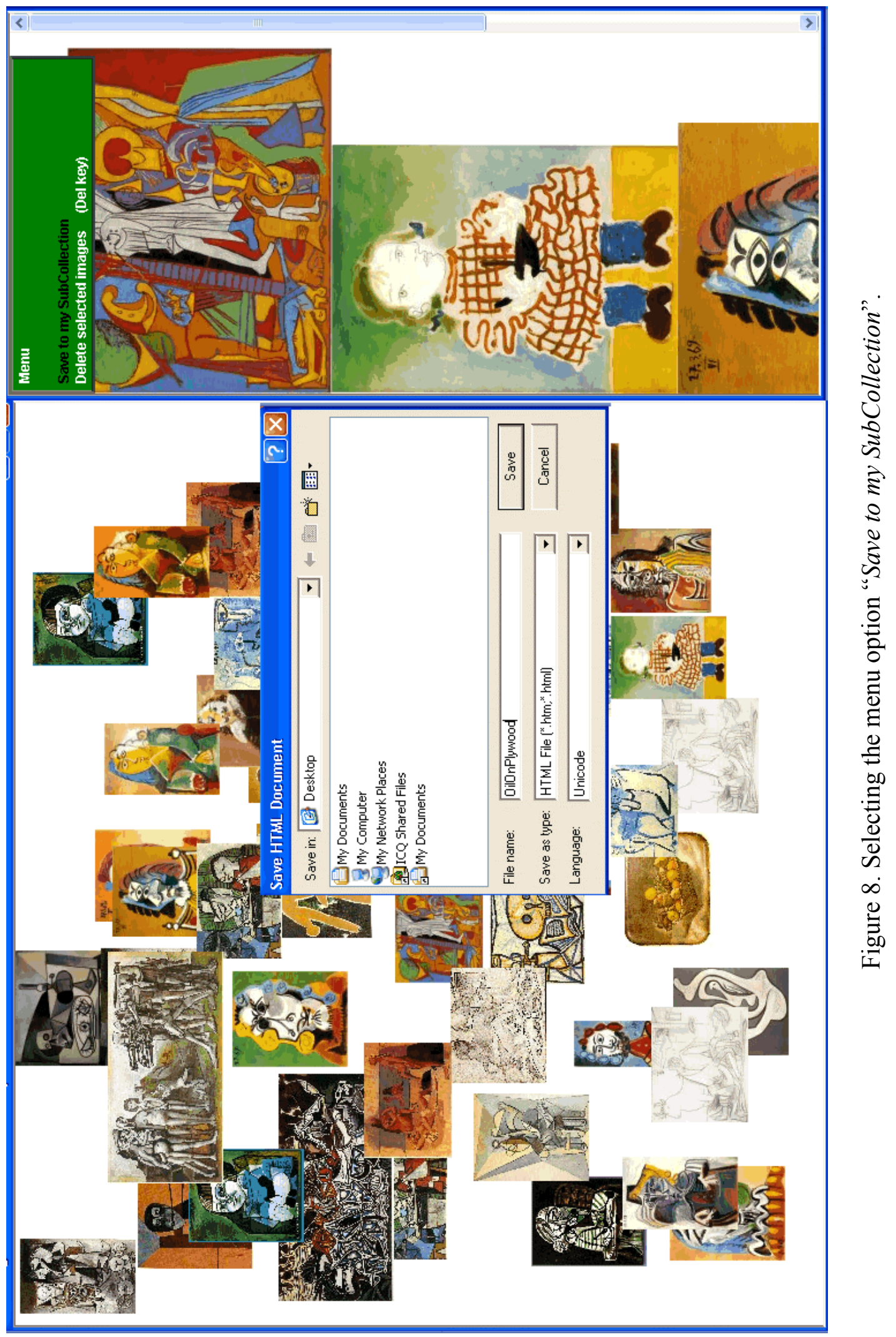


Visually expressing image collections using collages introduces a new approach for maximizing information learning. With indeterminacy from the collage display [Kerne 2001], the mind is motivated to creatively form associations among the image elements, thereby improving collection understanding. By providing a mechanism for users to gradually specify metadata values using the results from the collage display, users vary the content of the collage and visually learn what comprises the entire collection. Hence, users focus on the image elements first and then the metadata.

\section{Using an Ambient Slideshow for Collection Understanding}

Using an ambient slideshow allows users to select a subset of the collection for peripheral (or background) display. The images returned do not require a user's undivided attention or continuous real-time interaction [Mankoff et al. 2003]. Rather, they display in the background environment and engage the user's attention (through chance encounters) to learn about the artifact's membership in the collection. An ambient slideshow in the periphery of the environment slowly reveals the images in the collection and provides a simple mechanism to take a (possibly fleeting) look at the result set without requiring direct focus. Figure 9 shows an ambient display that slowly rotates new images into one of four frames on the display. 

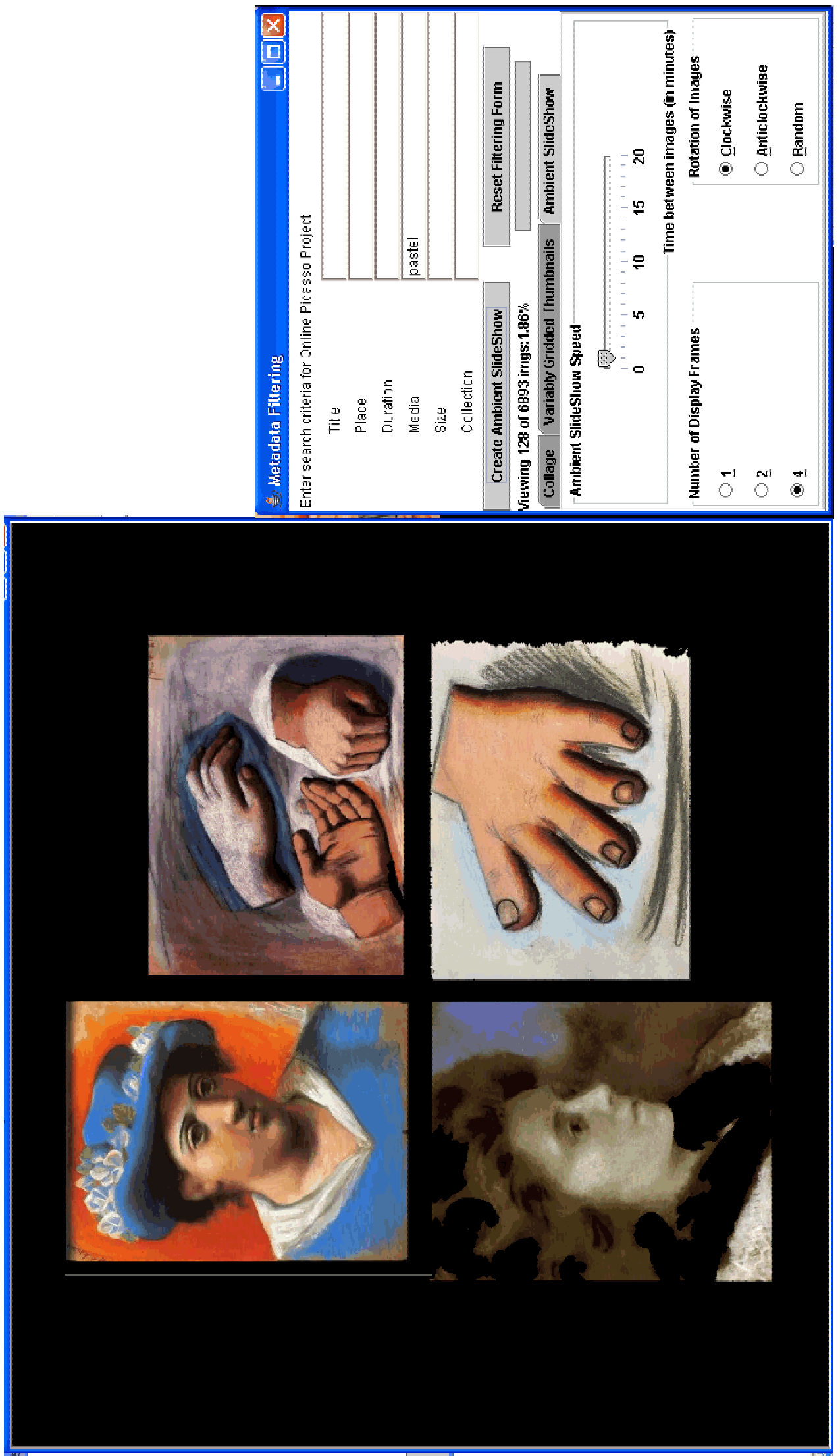

0
0
0
0
0
0
0
0
0
0
0
0
0
0
0
0
0
0 
When the collection becomes immersed in the environment in an ambient display, collection understanding will occur over longer periods of time (as predicted by research on incidental learning [Kelly et al. 2001]).

\section{Using Variably Gridded Thumbnails for Collection Understanding}

Current image collections use a grid of thumbnails as a direct manipulation interface to retrieve the original artifact. Clustering images based on size and orientation maximizes efficiency of screen space [Kang and Shneiderman 2000]. Thumbnails can also be categorized based on feature extraction similarities and text captions to aid users in finding images [Rodden et al. 2001]. By changing the default arrangement of the thumbnail images, users can quickly scan the resulting image set to see parallels among images in the collection.

Using variably gridded thumbnails, a relevance measure is returned for each image based on the number of matches found within a particular metadata field. Figure 10 shows artifacts from the Online Picasso collection where the media is specified as "lead pencil \& watercolor on paper" and the year as "1900". Any images completed in 1900 and containing lead, pencil or watercolor are returned with greater preference for images containing lead, pencil and watercolor. A color and a range of relevance values are stored for each bin. Images are placed into bins based upon their relevance value. Each grid element's background is colored to indicate the degree of relevance. Users can vary the number of bins to indicate the number of relevance categories for subdividing the images. 


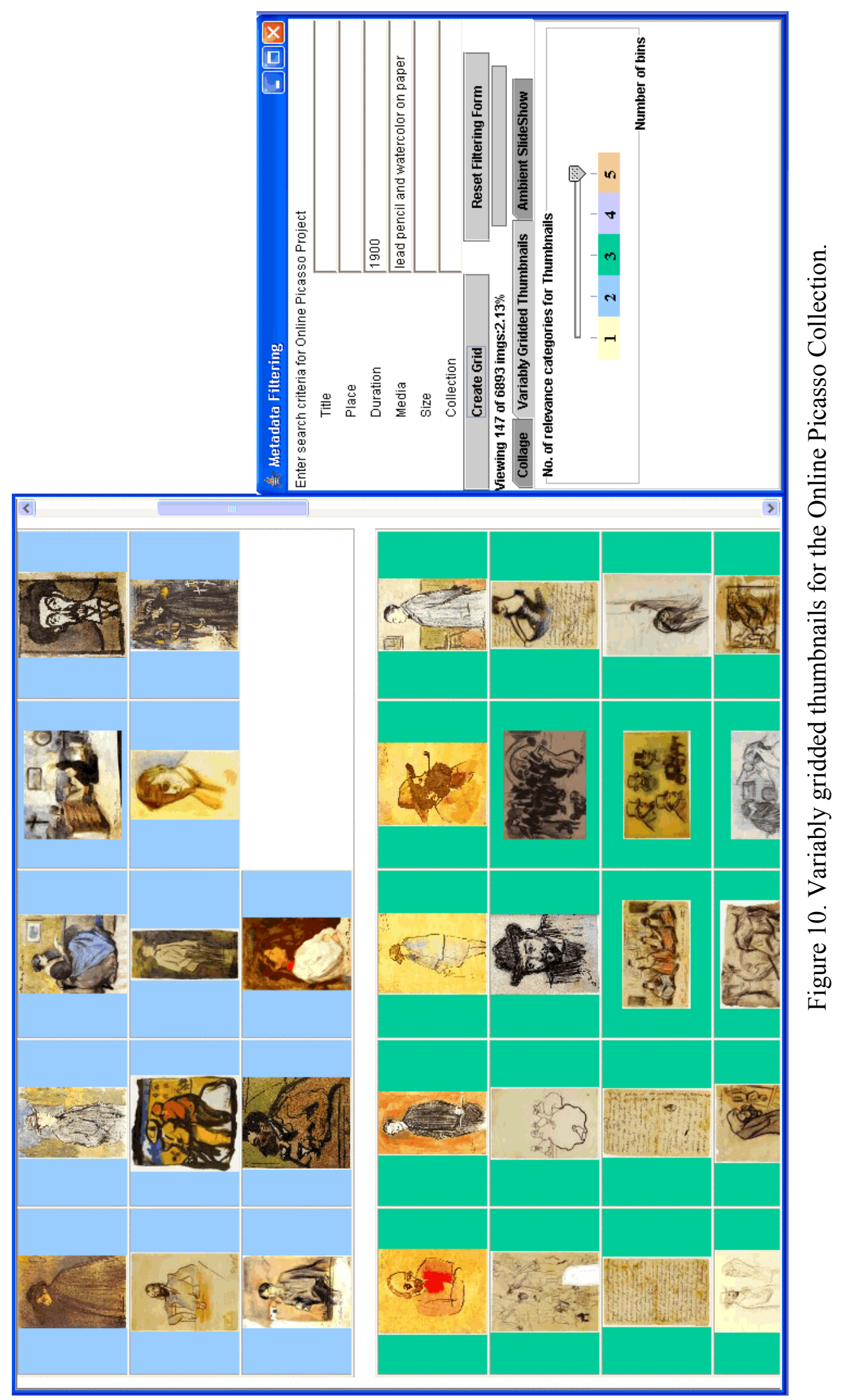


As illustrated in Figure 10, images placed on yellow backgrounds have the greatest match, with relevance decreasing when background colors are blue, green, purple and orange. In the future, this visualization will be used as a baseline to compare the streaming collage and ambient slideshow visualizations. 


\section{EVALUATION}

A usability study was conducted to assess the developed collection understanding visualization techniques based on the following factors: ease of use, time to come to an understanding of the collection and the visual appeal of the interface. Standard interaction design evaluation techniques as described by Preece, Rogers, and Sharp [Preece et al. 2002] were used.

As with any software tool, or indeed any artifact, the energy that went into the development of the functionality of the collection understanding tool would be wasted if users are not able to carry out their tasks because the user interface is too obtuse. The usability literature indicates that usability evaluations are typically better if conducted by professionals who have not been involved in the design and development of the product [Bias 1994]. Thus, I worked with usability experts at the University of Texas at Austin to design and conduct an independent evaluation of the emerging tool. A usability study of the intuitiveness of the streaming collage prototype was conducted and the findings are described below.

\section{Context}

The following describes a preliminary user test of the collection understanding tool. This is the first in a series of usability evaluations, ultimately to include further enduser testing in the lab with various user audiences, a heuristic evaluation [Nielsen 1994], field testing in a university classroom, and perhaps a usability walkthrough [Bias and Reitmeyer 1995]. 


\section{Participants}

The evaluators selected 15 graduate students from the University of Texas at Austin to participate in the preliminary study. None of the students who were recruited for the study had prior experience using the tool, nor did they have any formal training in art history or Picasso. The investigators recorded sessions through hand-written notes and video.

\section{Tasks}

After receiving a short scripted introduction, each participant was asked to begin working (without any training or direction) with the tool on his or her own for 10 minutes. During this period users were encouraged to "think aloud" and discuss their expectations [Lewis 1982]. At the completion of the 10-minute period, the participants each wrote a description of their comprehensive impression of the collection. Participants were instructed to write about the collection rather than the tool.

Participants were then asked to complete a set of tasks. The tasks were selected based on my description of a typical collection understanding experience and were those actions deemed critical to facilitating the proper use case scenario. The participants were encouraged to work without guidance except for periodic prompts from the experimenters to verbalize their thoughts as they worked. 


\section{Results}

The results of the initial tests indicate that users are not able to effectively "walkup and use" a majority of the tool's functions, however when given directed tasks they were more successful.

\section{No directed tasks}

During the phase in which they worked with no direction, more than half of the participants never created a collage with no filter values and an overwhelming majority attempted to type their own metadata values before ever beginning a collage. All of the participants attempted to type values into the search criteria fields at some point during test. Most users began the test by attempting to derive search criteria on their own based on their understanding of Picasso and his work. This left many users frustrated as their initial attempts at "querying the database" resulted in few if any returned results.

Even when users were able to get images to appear in the display space, many seemed hesitant to attempt to manipulate these images. Nearly half of the test subjects never right-clicked on images in the display space, thus never had an opportunity to review the metadata attributes for any images. Additionally, a third of the users never once attempted to manipulate images as they appeared in the display space and only two users who right-clicked on an image clicked on the terms in the menu to populate the search fields on the filtering form. 


\section{Directed tasks}

Users met with much more success when they were asked to complete discreet tasks. Upon being instructed to filter for images with no search criteria, all of the users were successful at creating a collage of the entire collection. Similarly, all but one of the test subjects successfully right-clicked on the images once instructed to view an image at full size.

\section{Discussion}

Based on the test results and feedback from the participants, we believe a few simple modifications to the tool's interface could enable users to work with the application more intuitively and shift focus from the application itself to active collection understanding. The most frequently observed and indicated problems involved three general areas of the interface: the user's initial engagement with the application, the metadata filtering form, and the collage space and controls.

Many participants were frustrated by the lack of an obvious starting point when asked to use the application on their own. Several remedies to this problem were suggested, including moving the "Create Collage" button from the "no man's land" under the filtering form to a location where it would be the first interface element the user came across, changing the label on the button (to "Start," perhaps), and automatically beginning a collage of the entire collection when the application is launched, thus providing users an explorable collage without requiring their input.

The problems most frequently experienced with the metadata filtering form dealt mostly with user confusion over what text would be appropriate for entry. Participants 
were particularly frustrated upon entering text that, by their estimation, matched the metadata field titles yet yielded no results. This is the typical frustration with IR-style interfaces mentioned earlier in the paper. Some participants felt the metadata field titles were far too vague to prompt their entry of good values. Recommended fixes to these problems include renaming the metadata fields and providing examples of metadata values present in the collection in an editable pull-down-style combo box for each metadata field. Additionally, one participant suggested that the presence of a region on the collage space to display the values of thumbnails, as they appear or as users mouse over them, would provide an introduction to the range of metadata values included in the collection. Clearly, the presence of the metadata fields on the right attracts the attention and action of novice users based upon their prior experience with IR-style interfaces.

Participants had some difficulty connecting the remote control portion of the metadata filtering window with its corresponding actions in the collage space. One participant noted that he would have understood these controls more quickly if they had been incorporated into the collage space itself. Another participant indicated that information now located in the metadata filtering window, such as application status and the number of images in the current collage and whole collection would be more visible as a component of the collage space.

While many participants expressed frustration with certain aspects of the application's interface, post-test discussions illustrated an excitement for the concept of collection understanding. This excitement seemed to fuel many of the participant's 
suggestions for enhancements and some users began to imagine different uses for the application.

Many participants reported that they enjoyed viewing a collection in this manner and felt the application enabled them to envision relationships among the images. One participant thought the collection tool helped her follow what she liked in the collection, even though she was not very familiar with Picasso's work.

Several participants were pleased that the application allowed them to create different collages with the same metadata, to clear the collage space and retain current metadata, and to make changes to the filtering form while the collage continued. Some participants noted that they appreciated the ability to control the speed of the collage; one in particular enjoyed viewing collages at the fastest possible speed. This participant imagined using the tool to browse the holdings of an online dealer of posters or photographs. He felt that this approach would be good for selling items shoppers do not seek out specifically.

Summary

These results indicate the current interface lacks intuitive controls and affordances for novice users, but that these users can quickly recover once they receive appropriate cues about how to properly manipulate the tool. These cues could easily be built into the interface design either through explicit instruction (e.g. help, or via an instructional mode) or more subtly by implying the phases of collection understanding in the interface. 


\section{FUTURE WORK}

The current usability study was largely formative [Gould and Lewis 1985; Gould et al. 1987] and tested only those intuitive interface attributes that were deemed essential to creating a collection understanding experience. The collection understanding tool will be modified to reflect the lessons learned from these studies.

Further studies can be done to compare the streaming collage interface with the Flamenco browser to determine whether users find one interface more helpful than the other and if externalizing the metadata alone is sufficient for collection understanding. In addition, preliminary studies can be done to uncover improvements that users would want based on their experience with current IR interfaces.

In the future, we will test a wider knowledge range of audiences. In addition, we have evaluated only the streaming collage prototype. Currently, the sub-collections interface is rudimentary and needs further design. A large number of artifacts collected would result in similar problems with scrolling through a large quantity of images. Hence visualizing sub-collections could involve creating clusters or collages with these results. These sub-collections can be linked with the original collection so that participants can further their analysis and use their sub-collections as starting points.

Usability studies on collection understanding using ambient slideshows and variably gridded thumbnails are yet to be conducted. The variably gridded thumbnails can be used as a secondary interface to the streaming collage once users come to their own understanding of the metadata values and what the metadata fields mean. Hence they would be able to define their own queries and further learn about particular image 
subsets. The variably gridded thumbnails interface can further probe the collection and return similar images clustered together. Usability studies can determine if the variably gridded thumbnails visualization is useful as a secondary tool for clustering the images and coming to a more thorough understanding of the collection.

Evaluating ambient displays is a challenge for the research community. My initial scenario for testing the Ambient Picasso slideshow would be to install this visualization on a plasma display in a busy hallway, videotape how people passing along the hallway interact with the display and determine at the end of an extended period of time (for instance two months) what impact, if any, did the display have on their thought processes about Picasso's works. Video recordings can be shown to refresh participants' minds about their initial reactions and engagement with the display. From this initial preliminary study, I would hope that participants experience a sense of curiosity and pleasant surprise about their serendipitous encounters with Picasso's works. I would also want participants to develop a desire to learn more about Picasso and suggest ways that the ambient display could facilitate this curiosity during their chance encounters with the Picasso collection. 


\section{CONCLUSION}

Collection understanding shifts the traditional focus of image retrieval in large collections from locating specific images to gaining a comprehensive view of the collection. Users visualize the collection using dynamic interaction to create their own understanding of the collection. As users narrow or expand their view, they acquire the general gist of why the artifacts belong to the collection and what characteristics the component elements share.

A streaming collage places all the images on one screen and removes the need for scrolling or clicking to a new page. Streaming collage varies the placement of the images on the screen in real time, creating different collages of the images in the collection. When users interactively filter the contents of the collection and perceive varying viewpoints of the artifacts, they are then inspired to derive their own relationships among the elements and come to a fuller understanding of the collection.

Variably gridded thumbnails change the order of display of the thumbnail images and help the user to focus their attention on images that may be more applicable. Resulting images that contain more matching metadata are displayed at the top of the screen to reflect higher relevance.

An ambient slideshow displays images on the periphery and does not explicitly draw the user's focus. Images reflected on the display change slowly over time and become embedded in the background environment. Chance glances to these ambient displays reveal images that were not previously known as a part of the collection. 
Through novel visualizations, users will be able to efficiently understand what specific collections have to offer. Collection insight will increase and enhance information access, making digital collections more valuable knowledge assets. In a world populated by thousands of digital libraries, a collection understanding tool can be used profitably by digital librarians and users alike to efficiently characterize and evaluate the holdings of various collections. 


\section{REFERENCES}

Apple iPhoto2. 2003. Commercial photo browser. Available at http://www.apple.com/iphoto/.

BALDONADO, M., AND WINOGRAD, T. 1997. SenseMaker: An informationexploration interface supporting the contextual evolution of a user's interests. In Proceedings of the SIGCHI Conference on Human Factors in Computing Systems. Atlanta, GA, ACM Press, 11-18.

BATES, M. 1989. The design of browsing and berrypicking techniques for the online search interface. Online Review 13, 5, 407-431.

BAUDISCH, P., AND GOOD N. 2002. Focus plus context screens: Displays for users working with large visual documents. In CHI '02 Extended Abstracts on Human Factors in Computer Systems. Minneapolis, MN, ACM Press, 492 - 493.

BEDERSON, B. 2001. PhotoMesa: A zoomable image browser using quantum treemaps and bubblemaps. In Proceedings of the 14th Annual ACM Symposium on User Interface Software and Technology. Orlando, FL, ACM Press, 71-80.

BIAS, R.G. 1994. The pluralistic usability walkthrough. In Usability Inspection Methods, Nielsen J. and Mack, R.L. Eds. John Wiley \& Sons, New York, NY.

BIAS, R.G., AND REITMEYER, P.B. 1995. Usability support inside and out. Interactions 2, 2, 29-32. 
BJORK, S. 2000. Hierarchical flip zooming: Enabling parallel exploration of hierarchical visualizations. In Proceedings of the Working Conference on Advanced Visual Interfaces. Palermo, Italy, ACM Press, 232 - 237.

BORNER, K., AND CHEN, C. 2002. Visual Interfaces to Digital Libraries, Lecture Notes in Computer Science (LNCS 2359). Springer Verlag, New York, NY.

CARD, S., MACKINLAY, J., AND SHNEIDERMAN, B. 1999. Readings In Information Visualization: Using Vision to Think. Morgan Kaufmann Publishers, San Francisco, CA.

CHRISTEL, M., HAUPTMANN, A., WACTLAR, H., AND NG, T. 2002. Collages as dynamic summaries for news video. In Proceedings of the Tenth ACM International Conference on Multimedia. Juan-les-Pins, France, ACM Press, 561 - 569.

COMBS, T., AND BEDERSON, B. 1999. Does zooming improve image browsing? In Proceedings of the Fourth ACM Conference on Digital Libraries. Berkeley, CA, ACM Press, 130 - 137.

Dublin core metadata element set, version 1.0: Reference description. 1998. Available at http://dublincore.org/documents/1998/09/dces/.

ELLIOTT, A. 2001. Flamenco image browser: Using metadata to improve image search during architectural design. In CHI '01 Extended Abstracts on Human Factors in Computer Systems. Seattle, WA, ACM Press, 69-70. 
ELLIOTT, A., HEARST, M., ENGLISH, J., SINHA R, SWEARINGEN, K., AND YEE, K. 2002. Finding the flow in website search. In Communications of the ACM. 45, 9, 42-49.

FOGARTY, J., FORLIZZI, J., AND HUDSON, S. 2001. Aesthetic information collages: Generating decorative displays that contain information. In Proceedings of the 14th Annual ACM Symposium on User Interface Software and Technology. Orlando, FL, ACM Press, 141-150.

FURNAS, G. 1986. Generalized fisheye views. In Proceedings of the SIGCHI Conference on Human Factors in Computing Systems. Boston, MA, ACM Press, 1623.

GOULD, J.D., BOIES S.J., LEVY, S., RICHARDS, J.T., AND SCHOONARD, J. 1987. The 1984 Olympic message system: A test of behavioral principles of system design. Communications of the ACM 30, 9, 758-769.

GOULD, J.D., AND LEWIS, C.H. 1985. Designing for usability: Key principles and what designers think. Communications of the ACM 28, 3, 300-311.

GRAHAM, A., GARCIA-MOLINA, H., PAEPCKE, A., AND WINOGRAD, T. 2002. Time as essence for photo browsing through personal digital libraries. In Proceedings of the Second ACM/IEEE-CS Joint Conference on Digital Libraries. Portland, OR, ACM Press, 326-335.

GREENBERG, S., AND ROUNDING, M. 2001. The notification collage: Posting information to public and personal displays. In Proceedings of the SIGCHI 
Conference on Human Factors in Computing Systems. Seattle, WA, ACM Press, 514521.

HOLMQUIST, L., AND SKOG, T. 2003. Informative art: Information visualization in everyday environments. In Proceedings of GRAPHITE 2003 International Conference on Computer Graphics and Interactive Techniques in Austalasia and South East Asia. Melbourne, Australia, ACM Press, 229-235.

HUANG, E., AND MYNATT, E. 2003. Semi-public displays for small, co-located groups. In Proceedings of the SIGCHI Conference on Human Factors in Computing Systems. Ft. Lauderdale, FL, ACM Press, 49-56.

ISHII H., AND BRYGG U. 1997. Tangible bits: Towards seamless interfaces between people, bits and atoms. In Proceedings of the SIGCHI Conference on Human Factors in Computing Systems. Atlanta, GA, ACM Press, 234-241.

ISHII, H., DAHLEY, A., AND WISNESKI, C. 1998. Water lamp and pinwheels: Ambient projection of digital information into architectural space. In Proceedings of the SIGCHI Conference on Human Factors in Computing Systems. Los Angeles, CA, ACM Press, 269-270.

ISHII, H., REN, S., AND FREI, P. 2001. Pinwheels: Visualizing information flow in an architectural space. In CHI '01 Extended Abstracts on Human Factors in Computer Systems. Seattle, WA, ACM Press, $111-112$. 
ISHII, H., WISNESKI, C., BRAVE, S., DAHLEY, A., GORBET, M., ULLMER, B., AND YARIN, P. 1998. ambientROOM: Integrating ambient media with architectural space. In Conference Summary of CHI 98. Los Angeles, CA, ACM Press, 18-23.

KANG, H., AND SHNEIDERMAN, B. 2000. Visualization methods for personal photo collections: Browsing and searching in the PhotoFinder. In Proceedings of IEEE International Conference on Multimedia and Expo. New York, NY, ACM Press, $1539-1542$.

KELLY, S.W., BURTON, M., KATO, T., AND AKAMATSU, S. 2001. Incidental learning of real-world regularities. Psychological Science 12, 1, 86-89.

KERNE, A. 1997. Collage machine: Temporality and indeterminacy in media browsing via interface ecology. In Proceedings of SIGCHI 1997 Extended Abstracts. Atlanta, GA, 297-298.

KERNE, A. 2000. Collage machine: An interactive agent of web recombination. Leonardo 33, 5, 347-350.

KERNE, A. 2001. Collage machine: Interest-driven browsing through streaming collage. In Proceedings of Cast01: Living in Mixed Realities. Bonn, Germany, 241-244.

KERNE, A. 2002a. Concept-context-design: A creative model for the development of interactivity. In Proceedings of the Fourth Conference on Creativity \& Cognition, an ACM SIGCHI International Conference. Loughborough UK, ACM Press, 192-199. 
KERNE, A. 2002b. Interface ecosystem, the fundamental unit of information age ecology. In Proceedings of SIGGRAPH02: Art and Animation. San Antonio, TX, 142-145.

KUCHINSKY, A., PERING, C., CREECH, M., FREEZE, D., SERRA, B., AND GWIZDKA, J. 1999. FotoFile: A consumer multimedia organization and retrieval system. In Proceedings of the SIGCHI Conference on Human Factors in Computing Systems. Pittsburgh, PA, ACM Press, 496-503.

LEWIS, C.H. 1982. Using the "thinking aloud" method in cognitive interface design. IBM Research Report RC-9265, 1982.

LI, J., AND SUN H. 2000. A virtual media (Vmedia) JPEG 2000 interactive image browser. In Proceedings of the Eighth ACM International Conference on Multimedia. Marina del Rey, CA, ACM Press, 501-502.

MALIK, J., BELONGIE, S., SHI, J., AND LEUNG, T. 2000. Contour and texture analysis for image segmentation. International Journal of Computer Vision 43, 1, 7 27.

MALLEN, E. 2004. The On-Line Picasso Project. Available at http://www.tamu.edu/mocl/picasso/.

MANKOFF, J., DEY, A., HSEIH G., KIENTZ, J., LEDERER, S., AND AMES, M. 2003. Heuristic evaluation of ambient displays. In Proceedings of the SIGCHI Conference on Human Factors in Computing Systems. Ft. Lauderdale, FL, ACM Press, 169-176. 
Merriam-Webster. Online Dictionary. 2004. Available at http://www.mw.com/home.htm.

MORAN, T., SAUND, E., VAN MELLE, W., GUJAR, A., FISHKIN, K., AND HARRISON, B. 1999. Design and technology for collaborage: Collaborative collages of information on physical walls. In Proceedings of the 12th Annual ACM Symposium on User Interface Software and Technology. Asheville, NC, ACM Press, 197-206.

NIELSEN, J. 1994. Heuristic Evaluation. In Usability Inspection Methods, Nielsen J. and Mack, R.L. Eds. John Wiley and Sons, Inc, New York, NY.

ONG, T., LEGGETT, J., WILSON, H., HATCH, S., AND REED, M. 2002. Interactive information visualization in the digital flora of Texas. In Visual Interfaces to Digital Libraries, Lecture Notes in Computer Science (LNCS 2359), Borner K. and Chen, C., Eds. Springer Verlag, New York, NY, 188-198.

PRASAD, B., GUPTA, S., AND BISWAS, K. 2001. Color and shape index for regionbased image retrieval. In Visual Form 2001, Lecture Notes in Computer Science (LNCS 2059), Arcelli C., Cordella L.P., Sanniti di Baja, Eds. Springer Verlag, New York, NY, 716-725.

PREECE J., ROGERS I., AND SHARP, H. 2002. Interaction Design: Beyond HumanComputer Interaction. John Wiley \& Sons, New York, NY.

RODDEN, K., BASALAJ, W., SINCLAIR, D., AND WOOD, K. 2001. Does organisation by similarity assist image browsing? In Proceedings of the SIGCHI 
Conference on Human Factors in Computing Systems. Seattle, WA, ACM Press, 190197.

SHNEIDERMAN B., FELDMAN, D., ROSE, A., AND GRAU X. 2000. Visualizing digital library search results with categorical and hierarchical axes. In Proceedings of the Fifth ACM Conference on Digital Libraries. San Antonio, TX, 57-66.

VAN LIERE, R., AND DE LEEUW, W. 1999. Exploration of large image collections using virtual reality devices. In Proceedings of the 1999 Workshop on New Paradigms in Information Visualization and Manipulation in conjunction with The Eighth ACM International Conference on Information and Knowledge Management. Kansas City, MO, ACM Press, 83-86.

WEISER, M., AND BROWN, J. 1995. Designing calm technology. Available at: http://www.ubiq.com/weiser/calmtech/calmtech.htm.

WITTEN, I., AND BAINBRIDGE D. 2002. How to Build a Digital Library. Morgan Kaufmann Series in Multimedia Information and Systems, Morgan Kaufmann Publishers Inc., San Francisco, CA.

WITTEN I., BODDIE, S., BAINBRIDGE, D., AND MCNAB, R. 1999. Greenstone: A comprehensive open-source digital library software system. In Proceedings of the fifth ACM conference on Digital Libraries. San Antonio, TX, 113-121.

WITTEN, I., MOFFAT, A., AND BELL, T. 1999. Managing Gigabytes: Compressing and Indexing Documents and Images. Morgan Kaufmann Publishers Inc., San Francisco, CA. 
YEE, K., SWEARINGEN, K., LI, K., AND HEARST M. 2003. Faceted metadata for image search and browsing. In Proceedings of the Conference on Human Factors in Computing Systems. Ft. Lauderdale, FL, ACM Press, 401-408.

ZHAI, S., SMITH, B.A., AND SELKER, T., 1997. Improving browsing performance: A study of four input devices for scrolling and pointing tasks. In Proceedings of INTERACT97: The Sixth IFIP Conference on Human-Computer Interaction. Sidney, Australia, ACM Press, 286-292. 
VITA

NAME:

PERMANENT ADDRESS:

EDUCATIONAL BACKGROUND:
Michelle T. Chang

11 Pierre Road

Felicity

Chaguanas

Trinidad W.I.

M.S. Computer Science Texas A\&M University College Station, Texas May 2004

B.S. Computer Science Angelo State University San Angelo, Texas May 2002 